\title{
Methodology to Predict Thermodynamic Data from Spectroscopic Analysis
}

ten Kate, Antoon J.B.; Gerretzen, Jan; van Manen, Henk-Jan; Kontogeorgis, Georgios M.; Bargeman, Gerrald

Published in:

Industrial \& Engineering Chemistry Research

Link to article, DOI:

10.1021/acs.iecr.0c04043

Publication date:

2020

Document Version

Peer reviewed version

Link back to DTU Orbit

Citation (APA):

ten Kate, A. J. B., Gerretzen, J., van Manen, H-J., Kontogeorgis, G. M., \& Bargeman, G. (2020). Methodology to Predict Thermodynamic Data from Spectroscopic Analysis. Industrial \& Engineering Chemistry Research, 59(49), 21548-21566. https://doi.org/10.1021/acs.iecr.0c04043

\section{General rights}

Copyright and moral rights for the publications made accessible in the public portal are retained by the authors and/or other copyright owners and it is a condition of accessing publications that users recognise and abide by the legal requirements associated with these rights.

- Users may download and print one copy of any publication from the public portal for the purpose of private study or research.

- You may not further distribute the material or use it for any profit-making activity or commercial gain

- You may freely distribute the URL identifying the publication in the public portal

If you believe that this document breaches copyright please contact us providing details, and we will remove access to the work immediately and investigate your claim 


\section{A methodology to predict thermodynamic data from}

\section{spectroscopic analysis}

Antoon J.B. ten Kate ${ }^{\dagger}$, Jan Gerretzen ${ }^{\dagger}$, Henk-Jan van Manen ${ }^{\dagger}$, Georgios M. Kontogeorgis ${ }^{\star}$, Gerrald Bargeman ${ }^{\dagger, *}$

$\dagger$ Nouryon Chemicals B.V., Research, Development \& Innovation, Zutphenseweg 10, P.O. Box 10, 7400 AA The Netherlands

\# Technical University of Denmark, Department of Chemical and Biochemical Engineering, Søltofts Plads, 2800 Kgs. Lyngby, Denmark

Key words: Gibbs excess energy; Spectroscopy; Predictive thermodynamics; Vapor-Liquid Equilibria

\section{ABSTRACT}

Sustainable processes, often dealing with complex mixtures, would benefit from the availability of more accurate and predictive thermodynamic models. Most existing models are (semi)empirical and require extensive input, while application to complex mixtures is cumbersome. In this work, the potential of extracting information about non-ideal behavior directly from spectroscopic information as sole source is studied. A methodology framework is proposed and 45 binary component mixtures with a broad non-ideality range were evaluated. Excess infrared absorbance spectra were successfully correlated with Gibbs excess energy using multivariate data analysis. For most binary mixtures experimental vapor liquid equilibrium literature data could be predicted accurately based on a model (UNIQUAC) using thermodynamic parameters obtained from the spectroscopic results. This also applied to binary mixtures that were not involved in the correlating step. Potential benefits of the investigated method are cost effective, accurate and quick measurement of non-ideality information, and improved predictive models, even for complex mixtures. The principle is demonstrated, and suggestions for further developments are provided.

\section{INTRODUCTION}

In recent decades there has been a clear trend in the chemical, pharmaceutical and materials industries to move towards more sustainable processes and new renewable products often based 
on molecules with (relatively) complex structures. Furthermore, to meet rapidly changing customer demands, there is a clear need for reduced time to market for these new products, and faster development and implementation of processes at manufacturing sites. Process simulation tools form one of the essential building blocks for process and product research and development. Currently available process simulation tools mostly rely on conventional thermodynamic models which were originally developed for the needs of the petrochemical industry. Existing models are often (semi)empirical and require a substantial amount of input data. Generation of input data is time-consuming and application of most existing models to complex mixtures of industrial interest is cumbersome. ${ }^{1}$ Consequently, there is a need for more accurate and predictive thermodynamic models and fast-track generation of required input data. To speed-up input data generation, several methodologies are under development.

Recently, the use of isothermal calorimetry (ITC) for the generation of thermodynamic parameters has been (re-)evaluated and reported. ${ }^{2-8}$ This methodology, sometimes in combination with molecular modelling, showed some successes in prediction of solid-liquid equilibrium ${ }^{2}$, liquidliquid equilibrium ${ }^{3}$, solvent selection for extraction ${ }^{4,5}$ and extractive distillation processes ${ }^{6,7}$, and in describing solvent interactions. However, for studies on acid - amine interactions thermodynamic parameters obtained from ITC measurements appeared to be concentration dependent, restricting their use to the evaluated concentration range only. ${ }^{8}$ Furthermore, the methodology appears to be suitable for mixtures consisting of strongly interacting molecules (such as acid-base interactions) but seems to be less accurate for mixtures with interaction enthalpies closer to $0 \mathrm{~J} \cdot \mathrm{mol}^{-1}$. Even more importantly, ITC provides mixing (molecular interaction) enthalpies only. This means that obtaining accurate thermodynamic parameters for process simulations from the ITC results requires either additional information on entropic effects, which are not obtained directly from the ITC measurements, or assumptions on the formation of adducts based on e.g. hydrogen bonding between molecules.

In recent decades spectroscopic methods have been investigated extensively as well. Preferential solvation could e.g. be determined from spectra showing changes in linewidth over different mole fractions using two-dimensional infrared spectroscopy (2D-IR) and nuclear magnetic resonance (NMR) spectroscopy. ${ }^{9}$ Furthermore, selection of aprotic solvents for optimal dye (Brooker's 
merocyanine) solvation resulted from a UV-Vis (ultraviolet - visible) spectroscopy study. ${ }^{10}$ Next to studying preferential solvation, spectroscopic data have also been related to activity coefficient models and vapor-liquid equilibria. Asprion et al. ${ }^{11,12}$ have used results from a comprehensive IR study for alcohol and phenol solutions in various solvents for fitting an "association" UNIQUAC model. Improved results as compared to original UNIQUAC results could be obtained. Jarisek et al. ${ }^{13}$ have proposed an approach to generate the activity coefficient for an organic (target) compound in a poorly described aqueous solution containing mixtures of organic molecules in very low concentrations. The concentration of the known target compound and water should be available and an assumption for the molality of the unknown organic molecules should be made as input for their methodology. Quantitative functional group information is obtained from ${ }^{13} \mathrm{C}$ NMR analysis, and subsequently mapped to groups used in the thermodynamic group contribution method. Based on this mapping, the activity coefficient for the target molecule is produced. ${ }^{13}$ Sassa and Katayama ${ }^{14,15}$ have used IR data for several alcohol solutions in the development of an associated Flory model. Activity coefficients calculated from the athermal associated solution theory, and self-association constants of alcohols and association constants between alcohol and solvent, both obtained separately from absorbance differences in infrared spectroscopy measurements, have been compared with activity coefficients based on VLE experiments. Ma et al. ${ }^{16}$ have used band frequency shifts in Raman spectra and a single activity coefficient value at infinite dilution to obtain Wilson parameters. Based on the obtained Wilson parameters they have been able to successfully predict the low-pressure VLE data for some highly non-ideal binary organic-aqueous systems. Xiao et al. ${ }^{17}$ and Xu et al. ${ }^{18}$ have used a similar approach as Ma et al. ${ }^{16}$ but have retrieved Wilson parameters from $\mathrm{C}-\mathrm{H}$ band shifts in IR spectra and ${ }^{1} \mathrm{H}$ NMR, respectively. Xu et al. ${ }^{19}$ have used the same approach, but instead of using the activity coefficient at infinite dilution they have used the azeotropic point as input for their model. Based on these studies several of these authors ${ }^{\text {e.g. }} 16$ have concluded that there must be some relation between spectroscopic and thermodynamic properties. However, they have not been able to show that thermodynamic parameters for non-ideality of the liquid phase can be obtained directly from analytical techniques without the need for additional information (such as the activity coefficient at infinite dilution). 
To the best of our knowledge only Xu et al. ${ }^{20}$ have derived thermodynamic parameters directly from spectroscopic ( ${ }^{1} \mathrm{H}$ NMR) measurements. In their methodology, they have generated activity coefficients using the Wilson equation ${ }^{21}$. The required Wilson parameters have been generated based on a simple semi-empirical physical model for the ${ }^{1} \mathrm{H}$-NMR shift as proposed by Deng et $a_{1} .^{22}$. In this model the required interaction energy difference between $\mathrm{j}-\mathrm{i}$ and $\mathrm{i}-\mathrm{i}$ pairs of molecules has been fitted from ${ }^{1} \mathrm{H}$ NMR chemical shift data, with the chemical shift of the pure component and that at infinite dilution as required as input parameters. Xu et al. ${ }^{20}$ have been able to accurately predict VLE data for 1-propanol-cyclohexane, 1-butanol-hexane and methanol-benzene mixtures over the entire mole fraction range. Zhu et al. ${ }^{23}$ have confirmed the validity of the approach for these alcohol-hydrocarbon mixtures but have also shown that the method is not generally valid for other mixtures, especially for more strongly non-ideal mixtures such as alcohol-water mixtures. Furthermore, they have shown that even when excellent fits for the chemical shift data can be obtained, this often results in poor VLE predictions (as e.g. seen for chloroform-acetone mixtures) and the results were different at differently selected wavelengths. Xu et al. ${ }^{24}$ agreed with the conclusions of Zhu et al. ${ }^{23}$ and explained that especially for water-alcohol mixtures ${ }^{1} \mathrm{H}$ NMR chemical shifts of the -OH group for the alcohol and for water overlap, making proper prediction of VLE impossible.

In our methodology we will show that it is possible to generate thermodynamic parameters and liquid phase non-ideality directly from spectroscopic techniques, with a focus on IR absorbance. Furthermore, we will show that within a family of mixtures (e.g. alcohol-ketone mixtures) VLE for a specific mixture can be successfully predicted based on excess absorbance data for that mixture and a model relating excess absorbance to excess Gibbs energy (the $A^{E}$ - $g^{E}$ model) for a limited number of mixtures within the family other than the mixture of interest. The purpose of the work described here is to demonstrate a first proof of principle for the hypothesized methodology. At the same time, we realize that the current contribution is only the start of this methodology development and several aspects deserve additional study. In the subsequent section our hypothesis and proposed methodology will be outlined in more detail.

\section{OUTLINE OF HYPOTHESIS AND PROPOSED METHODOLOGY}

\subsection{Hypothesis}


The underlying scientific assumption of the proposed methodology is that the complex intermolecular interactions present in diverse molecules and their mixtures can be captured by use of spectroscopic data and can be correlated to selected thermodynamic properties. The hypothesis is based on earlier publications as discussed in the introduction ${ }^{14-16}$ and our own observations that e.g. Mid-InfraRed (MIR) absorbance peaks change in intensity and position for binary mixtures of ethanol and heptane with different mixture compositions (see Fig. 1). If our hypothesis is valid, the spectral information can be used directly or indirectly and/or be translated into useful information which can result in (semi)predictive models for both low - and high-pressure applications, i.e. both activity coefficient models and equations of state, at least for screening purposes and potentially for quantitative parameterization as well.
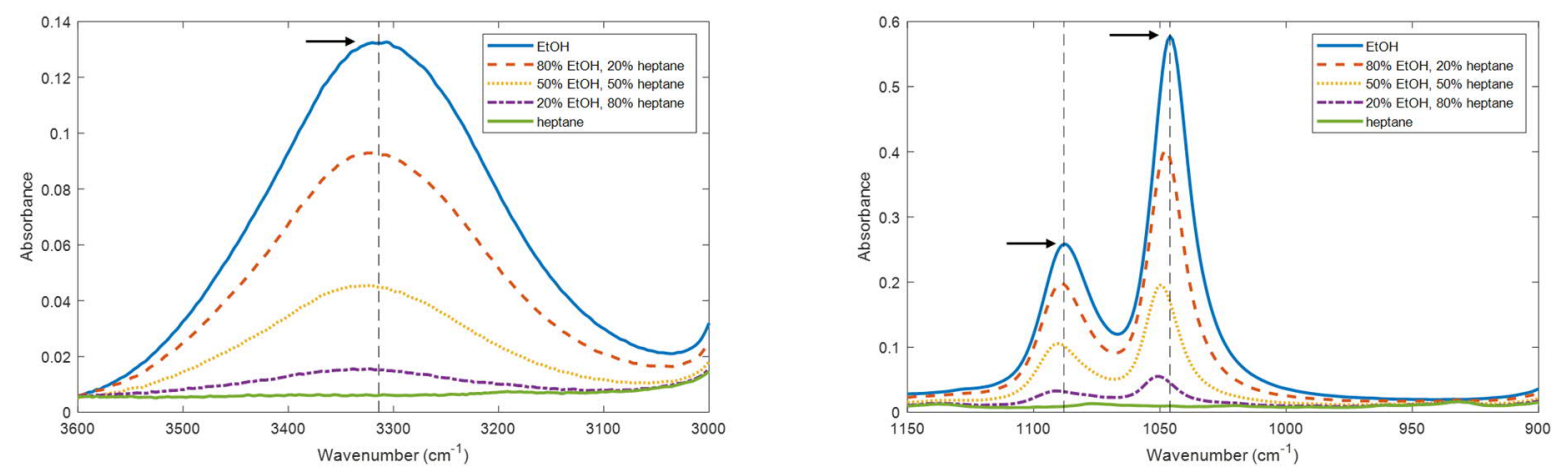

Figure 1. Shifts in MIR spectra for the binary system heptane - ethanol (EtOH). Left figure: blueshift (shift to higher frequency) in O-H stretch vibrations with decreasing EtOH concentration. Right figure: blueshift in asymmetric and symmetric C-O stretch vibrations with decreasing EtOH concentration.

\subsection{Proposed methodology}

The steps of the proposed (hypothesized) methodology are as follows (visualized in Fig. 2):

1. Spectra are measured for samples of varying concentration of the binary mixture of interest, including spectra of the pure components.

2. Excess absorbance data (see section 2.3) are derived from these absorbance spectra.

3. Excess energy data are predicted from the excess absorbance data, through the "spectralexcess energy' $\left(A^{E}-g^{E}\right)$ model.

4. The thermodynamic model, like UNIQUAC or SAFT, is parameterized using the predicted excess energy data. 
The parameterized thermodynamic model can then be used in typical applications, such as process design, solvent search or product design. Overall the method enables the direct prediction of nonideality in phase equilibria from (single phase) spectroscopic data only.

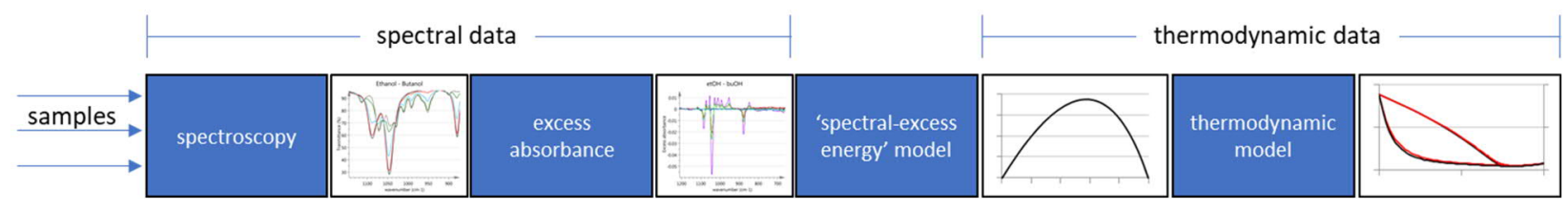

Figure 2. Schematic visualization of the proposed method for determination of phase equilibrium data from spectroscopic analysis.

The proposed methodology is described in detailed steps below, showing our considerations (hypotheses) for each step and the selected approach for providing a first proof of principle For the individual steps (spectroscopy; the excess absorbance - framework \& data-gathering; the spectral - excess energy model; the excess energy; the excess energy - data gathering; and the phase equilibrium). This approach is taken to make a clear distinction between hypotheses and the first proof of principle shown in this manuscript.

\section{Step 1: Spectroscopy}

Considerations: Depending on the spectroscopic technique used different molecular interactions are measured; hence it is expected that for a specific class of chemicals a specific spectroscopic technique is preferred, and this technique depends on the molecular interaction of concern within this class of chemicals. Simultaneous usage of multiple spectroscopic measurements (fusion) is expected to provide optimal results. The measurements should preferably cover various compositions and temperatures.

First proof of principle: Isothermal Mid-InfraRed (MIR) data is obtained at $25^{\circ} \mathrm{C}$.

Step 2: Excess absorbance - framework \& data-gathering

Considerations: Spectroscopic data provide information about composition as primary effect and about the molecular interactions as secondary effect. To distinguish the secondary from the primary effect, the approach of excess properties is taken. This concept is well established in thermodynamics, and it has also been used for excess spectra. ${ }^{25}$ Spectroscopic peaks can be 
changed in terms of amplitude, width, and position, and by using excess spectra such changes are considered.

First proof of principle: The excess absorbance is calculated from the difference of absorbance at a given wavelength as observed for the mixture minus that expected from an ideal mixture of pure components with the same composition at the same wavelength.

Step 3: Spectral - Excess energy model

Considerations: The objective of the model is to correlate the excess spectral data with the thermodynamic excess energy function. Preferably, the mathematical relation is based on fundamental understanding of the underlying physical and chemical phenomena, i.e. a mechanistic model. For example, it could be taken into consideration that the excess absorbance at a certain wavelength represents a certain amount of a certain type of energy. The opposite alternative is a data-driven model that is trained by the data to provide a correlation between excess spectral data and excess energy. The hybrid approach combines elements from both worlds, leading to an empirical relationship.

First proof of principle: Data-driven Partial Least Squares (PLS) regression with excess absorbance data at all wavelengths as independent variables and Gibbs excess energy as dependent variable, both at $\mathrm{T}=25^{\circ} \mathrm{C}$, is applied.

\section{Step 4a: Excess energy}

Considerations: The absorbance of light by a molecular mixture in spectroscopic analysis can be expressed in terms of energy. Similarly, the excess absorbance could be regarded an excess energy. However, it is unclear which type of excess energy function it relates to. In this study Gibbs excess energy $g^{E}$ has been used, but other energy functions like the closely related Helmholtz free energy $f^{E}$ may be appropriate as well. ${ }^{26}$ In subsequent work more focus on analysis of the energy terms is recommended.

First proof of principle: Gibbs excess energy at $25^{\circ} \mathrm{C}$ is used for first proof of principle.

Step 4b: Excess energy - data gathering

Considerations: The Gibbs excess energy is not directly measurable but is derived indirectly from thermodynamic data such as phase equilibria. The accuracy of the obtained Gibbs excess data 
depends on both the accuracy of the measurement of the mixture as well as of that of the pure constituents. The accurate determination of the excess energy requires accurate measurement, and even then, may be hampered by difficult analysis if the pure constituents exhibit substantially different volatility (i.e. for wide-boiling systems).

First proof of principle: Gibbs excess energies are estimated using UNIFAC (Aspenplus version 10), resolving the issue of data availability and inaccuracy for systems comprising constituents of different volatility.

Step 5: Phase equilibrium

Considerations: Thermodynamic models can be parameterized using the derived Gibbs excess energy data from excess spectral data. Preferably the data cover a broad range of compositions at various temperatures. Then, the parameterized thermodynamic model can be used to predict the phase equilibrium to be studied. Established thermodynamic models can be used, like the $g^{E}$ models such as NRTL or UNIQUAC, or suitable equations of state (EoS).

First proof of principle: Binary parameters $\mathrm{B}_{\mathrm{ij}} / \mathrm{B}_{\mathrm{ji}}$ of UNIQUAC (Aspenplus version 10) have been regressed using predicted Gibbs excess energy data at $25^{\circ} \mathrm{C}$.

\subsection{Excess concepts for thermodynamic and spectroscopic properties}

In an ideal mixture of components each component obeys Raoult's law for all compositions. This applies especially for components having a similar structure. However, many binary mixtures behave non-ideally, meaning that mixture properties are not proportionally related to their molar mixing ratio. This non-ideality stems from changes in intermolecular and intramolecular interactions due to mixing of the two pure components. Between components a broad range of possible interactions may occur, such as hydrogen bonding, dipole-dipole interactions, and dipoleinduced dipole interactions (see e.g. Israelachvili ${ }^{27}$ for more information about molecular interactions).

To describe and explain non-ideal behavior excess properties are widely used. ${ }^{28-31}$ These excess properties are defined as:

$$
M^{E}=M-M^{\text {id }}
$$


where $M^{E}$ is the value of the excess property of interest, $M$ is the value of the property of the actual solution, and $M^{i d}$ is the value of the property of the ideal solution at the same temperature, pressure, and composition. ${ }^{32}$

$M^{i d}$ is calculated as the molar average of $M$ of the constituents in their pure form:

$$
M^{\text {id }}=\sum x_{i} M_{i}^{\text {pure }}
$$

The most broadly used excess properties are the Gibbs excess energy $\left(g^{E}\right)$, excess enthalpy $\left(h^{E}\right)$, and excess entropy $\left(s^{E}\right){ }^{33,34}$ It should be noted that eq. (2) applies to the excess enthalpy only. Because of the earlier suggested relation between spectroscopic data and thermodynamic information ${ }^{14-16}$ we hypothesize that excess spectroscopy can be used to study non-ideality and be related to excess thermodynamic data. The concept of excess spectroscopy (or excess absorbance) is comparable to the concept of excess functions in thermodynamics. The excess spectrum $A^{E}$ describes the non-ideal contribution of the spectrum for a mixture, and can be obtained from subtracting the 'ideal' spectrum of a binary mixture $A^{i d}$ from the measured spectrum of the binary mixture $A$, according to:

$$
A^{E}=A-A^{i d}
$$

If we denote the spectrum of a pure component $\mathrm{i}$ as $A_{i}$ and the spectrum of the other pure component $\mathrm{j}$ as $A_{j}$, the ideal spectrum $A^{i d}$ can be calculated according to:

$$
A^{i d}=x_{i} \cdot A_{i}+x_{j} \cdot A_{j}
$$

where $x_{i}$ and $x_{j}$ represent the molar fractions of the pure components in the binary mixture.

This approach has been proposed by Corsetti et $\mathrm{al}^{35}$. Another approach to determine the excess absorbance spectra is based on using molar absorption coefficients (i.e. molarity corrected) as proposed by Li et al. ${ }^{25}$. Since the $A^{E}-g^{E}$ model results for individual mixtures of chloroformheptane and ethanol-heptane were similar for both approaches, the approach proposed by Corsetti et $a l .{ }^{35}$ was used for our proof of principle work. The resulting excess spectrum shows the absorption of (e.g. infrared) light due to non-ideal effects. In principle the excess absorbance encompasses both changes in peak amplitude and peak position (peak shift). In the current study aiming at proof of principle the excess absorbance was calculated over the entire wavenumber 
range, resulting in excess absorbance spectra. When using Partial Least Squares (PLS) as bilinear regression technique, this provides good solutions for systems where peak amplitude differences are dominant compared to peak shifts. It is known that strong peak shifts may have a negative effect on the performance of PLS regression ${ }^{36,37}$. Therefore, for systems where peak shifts play the most important role (such as for NMR spectroscopy), conventional PLS regression using excess spectra may not provide the most optimal solution. The concept of excess spectra has been studied earlier, e.g. by Li et al. ${ }^{25}$ where new insights were unveiled on hydrogen bonding in tertbutanol $-\mathrm{CCl}_{4}$ mixtures using excess spectroscopy, but to the best of our knowledge has never been related directly to excess thermodynamic properties such as Gibbs excess energy.

\subsection{Creating the 'excess absorbance-Gibbs excess energy $\left(A^{E}-g^{E}\right)$ ' model link}

In general, at a high level, three different modeling approaches can be distinguished:

- Mechanistic modeling

- Data-driven modeling

- Hybrid modeling

Mechanistic models are based on fundamental physical and chemical relationships, hence offer a consistent framework with predictive power. However, their development requires rigorous insight and proper parameterization, which can be effort-demanding and time-consuming. Data-driven models are based on relationships or patterns within dataset(s). In principle, they do not make use of other information such as a-priori knowledge. Hybrid models are based on the combination of mechanistic and data-driven models.

As the purpose of the study was to show a first proof of principle that spectroscopic techniques can provide suitable information on mixture non-ideality, multivariate linear regression models (data-driven models) have been used to link excess absorbance to the Gibbs excess energy. It should be noted that other (mechanistic or hybrid) models might be used as well and the multivariate linear regression model is only used for illustration purposes. Partial Least Squares (PLS) regression is the model chosen in this work, since it is commonly applied for analyzing and modeling spectroscopic data. It aims to construct a linear model of the form:

$$
\boldsymbol{y}=a+B \boldsymbol{X}
$$


where in our case $\boldsymbol{y}$ represents the vector of Gibbs excess energy $\left(g^{E}\right)$ values, $\boldsymbol{X}$ the matrix of excess absorbance spectra $\left(A^{E}\right), a$ the intercept, and $B$ the vector of regression coefficients (one for each wave number in the excess absorbance spectra).

The commonly used least squares solution to obtain $a$ and $B$ does not work in this case, because the number of wavenumbers (variables) in $\boldsymbol{X}$ far exceeds the number of spectra, the so-called "curse of dimensionality" ${ }^{38}$ Moreover, neighboring variables in $\boldsymbol{X}$ are highly correlated due to the spectral nature of the data. PLS (partial least squares) regression tries to overcome both issues via a dimension reduction step, where matrix $\boldsymbol{X}$ is decomposed into new orthogonal (i.e. uncorrelated) so-called latent variables (LVs). Only a few LVs are commonly required to accurately "describe" the variation in $\boldsymbol{X}$, thereby eliminating the "curse of dimensionality" since the number of LVs is typically lower than the number of spectra. By performing the linear regression step in the reduced space of $\boldsymbol{X}$ a linear model is obtained, which is later transformed back to the original space of $\boldsymbol{X}$ to obtain the final model $\boldsymbol{y}=a+B \boldsymbol{X}$.

Root Mean Square Error (RMSE) values have been used to judge the validity of correlating excess absorbance with Gibbs excess energy. The RMSE is defined as:

$$
R M S E=\sqrt{\frac{(y-\hat{y})^{2}}{n}}
$$

where $y$ represents the true $g^{E}$ value, $\hat{y}$ the predicted $g^{E}$ value by the PLS model, and $n$ the number of spectra per model.

Many more details about PLS regression can be found in dedicated subject matter handbooks or review articles such as references. ${ }^{38-41}$

\section{METHODS}

\subsection{Data gathering spectroscopy}

Purities and suppliers of chemicals used in the experimental program are specified in Table 1. All chemicals were analytical grade and reported purities are based on supplier information and have not been validated. Chemicals have been used without further purification. In total, 45 different binary mixtures (see Tables 2 and 3) have been examined to make a first assessment of the 
performance of the proposed methodology. For each of the binary mixtures, 30 different samples varying in molar composition ratio have been prepared using a robotic platform from Bosch Packaging Technologies, consisting of two Stäubli six-axis robotic arms and a gravimetric dispensing formulation station. The 21 molar composition setpoints provided to the robot for the binary mixtures used for calibrating of the model ranged from 0\%:100\% to 100\%:0\% with 5\% interval steps. The 9 molar composition setpoints provided to the robot for the preparation of the binary mixtures used for validation of the model were $97.5 \%: 2.5 \%, 85.625 \%: 14.375 \%$, 73.75\%:26.25\%, $\quad 61.875 \%: 38.125 \%, \quad 50 \%: 50 \%, \quad 38.125 \%: 61.875 \%, \quad 26.25 \%: 73.75 \%$, 14.375\%:85.625\%, and 2.5\%:97.5\%. For all experiments the mixture compositions obtained deviated less than $0.1 \%$ (absolute) from the composition provided as input to the robot. The platform has been controlled via Batch Management System (Bosch) software. Mid-InfraRed (MIR) spectra have been recorded for all samples using a Perkin-Elmer Spectrum 100 FT-IR instrument, equipped with a Golden Gate diamond ATR accessory. Spectra have been acquired in the wavenumber range of $500-4000 \mathrm{~cm}^{-1}$, with a resolution of $4 \mathrm{~cm}^{-1}$ and an average of 16 scans. Additionally, all samples have been measured using NIR (Near-InfraRed) and Raman spectroscopy. Since the focus in this paper is on proof of principle, MIR has been selected as preliminary analysis based on indications that this type of spectroscopy gave suitable results to predict $g^{E}$ data. Experiments with NIR and Raman gave similar results (see Supporting Information for illustration purposes), albeit generally with slightly lower accuracy. However, our studies were too limited to exclude other spectroscopic techniques like NMR, NIR or Raman spectroscopy, or the combination thereof (data fusion) as proper source of spectral data for the prediction of the excess energy function (like $g^{E}$ ).

Table 1. Specification of chemicals used in the experimental program.

\begin{tabular}{|c|c|c|}
\hline Chemical Name & Purity specified by the supplier & Supplier \\
\hline Heptane & $>99 \%$ & Acros \\
\hline Nonane & $>99 \%$ & Merck \\
\hline Undecane & $>99 \%$ & Acros \\
\hline Toluene & $>99 \%$ & JT Baker \\
\hline Chloroform & $>99 \%$, stabilized with $0.25 \%$ ethanol & JT Baker \\
\hline Ethanol & $100 \%$ & Acros \\
\hline 1-Butanol & $99 \%$ & \\
\hline
\end{tabular}




\begin{tabular}{|c|c|c|}
\hline 1-Hexanol & $>99 \%$ & Acros \\
\hline Methyl Ethyl Ketone (MEK) & $99.5 \%$ & JT Baker \\
\hline 2-Hexanone & $98 \%$ & Acros \\
\hline 2-Octanone & $>99 \%$ & Acros \\
\hline Acetic Acid & $99-100 \%$, glacial & DI tap water \\
\hline Water & DI tap water & Acros \\
\hline n-Butyl Amine & $>99 \%$ & Sigma-Aldrich \\
\hline 1-Hexene & $97 \%$ & \\
\hline
\end{tabular}

Table 2. An overview of all binary mixtures that have been studied using spectroscopic methods and used in the individual mixture $A^{E}-g^{E}$ model approach.

\begin{tabular}{|l|c|c|c|}
\hline \multicolumn{3}{|c|}{ Mixtures with different functional groups used in constructing the individual } \\
\hline chloroform - heptane & acetic acid - water & toluene - MEK & heptane - 1-butylamine \\
\hline chloroform - ethanol & acetic acid - ethanol & toluene - ethanol & heptane - ethanol \\
\hline chloroform - 1-butanol & acetic acid - MEK & toluene - 1-butanol & ethano - MEK \\
\hline chloroform - MEK & acetic acid - heptane & toluene - 1-hexene & ethanol - water \\
\hline chloroform - toluene & acetic acid - toluene & toluene - heptane & \\
\hline chloroform - acetic acid & & & \\
\hline
\end{tabular}

The 1,350 MIR spectra obtained for 45 mixtures with 30 different molar compositions each have been visually checked for outliers using Principal Component Analysis (PCA). The first two Principal Components (PCs) generally described almost all variation in the spectra of each binary system and showed a clear pattern in the scores. Any deviation from that pattern may indicate an outlying spectrum, for example due to suboptimal contact with the ATR crystal. The spectra that have been marked as outliers according to this procedure have been remeasured. Data from the wavenumber range $600-4000 \mathrm{~cm}^{-1}$ have been used and the excess spectra have been preprocessed via mean-centering, as is customary for virtually all PLS models. No additional preprocessing methods have been applied. For each PLS model the number of selected LVs has been optimized via 10 -fold cross-validation.

Table 3. An overview of all binary mixtures that have been studied using spectroscopic methods and used in the mixture family $A^{E}-g^{E}$ model approach. 


\begin{tabular}{|c|c|c|c|}
\hline Mixture family & Mixture & $\begin{array}{c}\text { Mixture used in } \\
\text { constructing the family } \\
A^{E}-g^{E} \text { model }\end{array}$ & $\begin{array}{c}\text { Mixture used in } \\
\text { validation of the family } \\
A^{E}-g^{E} \text { model }\end{array}$ \\
\hline \multirow{9}{*}{ alcohol-ketone } & ethanol - MEK & $\mathrm{x}$ & \\
\hline & ethanol - 2-hexanone & & $\mathrm{x}$ \\
\hline & ethanol - 2-octanone & $\mathrm{x}$ & \\
\hline & 1-butanol - MEK & & $\mathrm{x}$ \\
\hline & 1-butanol - 2-hexanone & & $\mathrm{x}$ \\
\hline & 1-butanol - 2-octanone & & $\mathrm{x}$ \\
\hline & 1-hexanol - MEK & $\mathrm{x}$ & \\
\hline & 1-hexanol-2-hexanone & & $\mathrm{x}$ \\
\hline & 1-hexanol - 2-octanone & $\mathrm{x}$ & \\
\hline \multirow{9}{*}{ alkane-ketone } & heptane-MEK & $\mathrm{x}$ & \\
\hline & heptane - 2-hexanone & & $\mathrm{x}$ \\
\hline & heptane - 2-octanone & $\mathrm{x}$ & \\
\hline & nonane - MEK & & $\mathrm{x}$ \\
\hline & nonane - 2-hexanone & & $\mathrm{x}$ \\
\hline & nonane -2-octanone & & $\mathrm{x}$ \\
\hline & undecane - MEK & $\mathrm{x}$ & \\
\hline & undecane - 2-hexanone & & $\mathrm{x}$ \\
\hline & undecane - 2-octanone & $\mathrm{x}$ & \\
\hline \multirow{9}{*}{ alkane-alcohol } & heptane - ethanol & $\mathrm{x}$ & \\
\hline & heptane - 1-butanol & & $\mathrm{x}$ \\
\hline & heptane - 1-hexanol & $\mathrm{x}$ & \\
\hline & nonane - ethanol & & $\mathrm{x}$ \\
\hline & nonane - 1-butanol & & $\mathrm{x}$ \\
\hline & nonane - 1-hexanol & & $\mathrm{x}$ \\
\hline & undecane - ethanol & $\mathrm{x}$ & \\
\hline & undecane - 1-butanol & & $\mathrm{x}$ \\
\hline & undecane - 1-hexanol & $\mathrm{x}$ & \\
\hline
\end{tabular}

\subsection{Data gathering Gibbs excess energy}

For sake of a first demonstration of the proposed methodology to derive quantitative information about liquid non-ideality from spectroscopic data only, Gibbs excess energy $\left(g^{E}\right)$ has been chosen 
as excess energy function. The main reason is the accessibility of $g^{E}$ data and the direct link between $g^{E}$ and activity coefficients, hence, to phase equilibria that are relevant for chemical engineering applications. In future work the appropriate excess energy function should be further investigated. As an alternative for the Gibbs excess energy, the excess Helmholtz energy $f^{E}$ might provide an adequate energy function. ${ }^{26}$ It should be noted that Gibbs free energy and Helmholtz free energy are closely related and can be converted into each other. ${ }^{42}$

The Gibbs excess energy is not directly measured, but can be derived from VLE, SLE, and LLE data. Typically, the binary interaction parameters of the $g^{E}$ model are regressed to experimental phase equilibria data comprising composition, pressure and temperature. With the parameterized $g^{E}$ model the Gibbs excess energy can be calculated for given composition and conditions. When parameterized for all constituting binary subsystems, $g^{E}$ models like UNIQUAC and NRTL can also predict the behaviour of multi-component systems.

It should be noted that the existing local-composition based $g^{E}$ models are in fact semi-empirical relations to describe the non-ideality. They have proven their strength in representing the nonideality to a sufficient quantification level to be used effectively in process and product design. ${ }^{43}$

The indirect way of determining the Gibbs excess energy may pose a problem for systems where the primary effects are dominant. For example, in a binary system of wide-boiling constituents, a deviation from ideality may not have a significant effect on the phase equilibrium, hence the deviation from ideality may not be visible from the composition, pressure and temperature data. In those cases, the regression might lead to the impression that the liquid is ideal, even though it is not. For the representation of the phase equilibria for process and product design, this is acceptable; however, for providing training data about the Gibbs excess energy models it is a concern.

An alternative approach is using estimation methods, like UNIFAC and COSMO-RS. Being predictive methods for the phase equilibrium they are likely to be less accurate, however they provide information about the liquid non-ideality irrespective of the other system characteristics. For the purpose of this manuscript, original UNIFAC (Aspenplus, version 10) ${ }^{44-47}$ has been chosen 
as method to generate the Gibbs excess energy data, for the reason described as well as for their general applicability, and consequently the data availability, for all systems considered in this study. On the other hand, it is recognized that it has the drawback of using an estimation method for setting up another prediction method, yet for proof of principle it is considered acceptable. It is also recognized that the establishment of appropriate datasets of the excess energy function is a key element for further development of the methodology. For the purpose of generating the $A^{E}-g^{E}$ models, Gibbs excess energy data at ambient temperature were generated and used to calibrate the model, since the excess absorbances have been measured at ambient temperature only.

\subsection{Individual $A^{E}-g^{E}$ models and family $A^{E}-g^{E}$ models}

In our proof of principle work several $A^{E}-g^{E}$ models have been constructed. For 21 binary mixtures consisting of two components with different functional groups (see Table 2) individual $A^{E}-g^{E}$ models have been constructed based on 21 different compositions of the individual mixture (see section 3.1). The obtained individual $A^{E}-g^{E}$ model has been validated with 9 other compositions of the same mixture components (see section 3.1). This procedure has been followed to validate whether there is liquid non-ideality information in spectral data.

Furthermore, family $A^{E}-g^{E}$ models have been constructed and validated for three different mixture families (the alcohol-ketone family, the alkane-ketone family, and the alkane-alcohol family, see Table 3). A mixture family is defined as a group of binary mixtures in which the binary constituents are selected from two different classes of chemicals. All individual families consist of 9 mixtures formed by 3 chemicals from one chemical class (sharing a specific functional group and having varying chain-lengths), and 3 chemicals from another chemical class (sharing another functional group and having varying chain-length). The 21 calibration compositions (see section 3.1 for compositions) of the "extremes" of a family (i.e. the 4 binary mixtures with the longest and shortest chains, see Table 3 and Fig. 3 (top) as example for the alkane-ketone family) have been used to construct the family $A^{E}-g^{E}$ model for a specific family. The validation compositions of the other binary mixtures of the family (not used in the construction of the family $A^{E}-g^{E}$ model) have been used to validate the constructed family $A^{E}-g^{E}$ model. This procedure has been followed to validate the predictive power of the $A^{E}-g^{E}$ model within a mixture family. 
Combined family $A^{E}$ - $g^{E}$ models for the alcohol-ketone \& alkane-ketone \& alkane-alcohol combined family and the alcohol-ketone $\&$ alkane-ketone combined family have been constructed as well. For these two combined families the 21 calibration compositions (see section 3.1 ) of the "extremes" of a combined family (i.e. the 4 binary mixtures with the longest and shortest chains per individual family, see Table 3 and Fig. 3 (bottom) as example for the alcohol-ketone \& alkaneketone family), have been used to construct the combined family $A^{E}-g^{E}$ model for a specific combined family. The validation compositions of the other binary mixtures of the combined family (not used in the construction of the family $A^{E}-g^{E}$ model) have been used to validate the constructed family $A^{E}-g^{E}$ model. This procedure has been followed to validate the predictive power of the $A^{E}$ $g^{E}$ model within a combined mixture family.
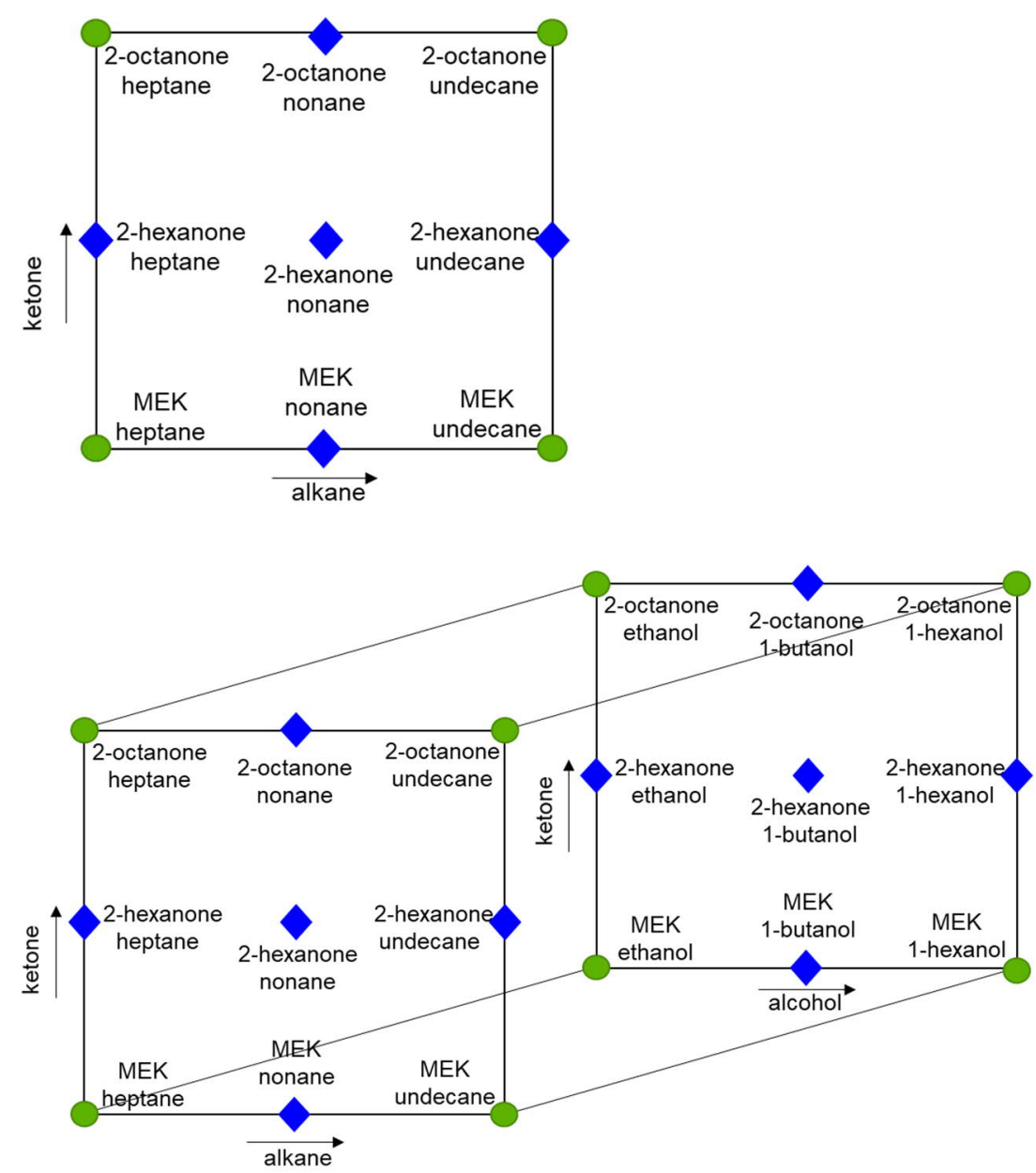

Figure 3. Model setup for the "alkane-ketone" family (top) and for the "alkane-ketone \& alcoholketone" combined family (bottom). The green circles represent the calibration set (based on which the $A^{E}-g^{E}$ model is developed) of the (combined) family model, while the blue diamond symbols represent the validation set (based on which the $A^{E}-g^{E}$ model performance is assessed).

\subsection{Modelling VLE}


Based on the generated $A^{E}-g^{E}$ models, Gibbs excess values have been predicted for all mixtures and mixture families for all mixture compositions evaluated through MIR. These predicted Gibbs excess values were used as 'pseudo'-data to regress the binary interaction parameters $\mathrm{B}_{\mathrm{AB}}$ and $\mathrm{B}_{\mathrm{BA}}$ of the respective binary systems in UNIQUAC in Aspen version 10. These binary interaction parameters are related to the activity coefficient $\gamma_{i}$ according to the following set of equations ${ }^{48}$, which are based on Abrams and Prausniz: ${ }^{49}$

$$
\ln \gamma_{i}=\ln \frac{\varphi_{i}}{x_{i}}+\frac{z}{2} q_{i} \ln \frac{\theta_{i}}{\varphi_{i}}-q_{i} \ln \sum_{k}\left(\theta_{k} \tau_{k i}\right)-q_{i} \sum_{j}\left(\theta_{j} \tau_{i j} / \sum_{k}\left(\theta_{k} \tau_{k j}\right)\right)+l_{i}+q_{i}-\frac{\varphi_{i}}{x_{i}} \sum_{j}\left(x_{j} l_{j}\right)
$$

$$
\theta_{i}=q_{i} x_{i} / \sum_{k}\left(q_{k} x_{k}\right)
$$

$$
\varphi_{i}=r_{i} x_{i} / \sum_{k}\left(r_{k} x_{k}\right)
$$

$$
l_{i}=\frac{z}{2}\left(r_{i}-q_{i}\right)+1-r_{i} \text { with } z=10
$$

$$
\ln \left(\tau_{i j}\right)=B_{i j} / T
$$

$$
r_{i}=\sum_{k}\left(v_{k}^{i} R_{k}\right)
$$

$$
q_{i}=\sum_{k}\left(v_{k}^{i} Q_{k}\right)
$$

The group volume parameter $R_{k}$ and the group area parameter $Q_{k}$ are calculated according to Bondi et al. ${ }^{50}$

This procedure has been followed for all individual mixtures in the individual mixture approach as well as for all mixtures involved in the (combined) family approach. Subsequently, with the UNIQUAC model parameterized in this way the activity coefficients $\left(\gamma_{i}\right.$ and $\left.\gamma_{j}\right)$ and vapor-liquid equilibria have been predicted (assuming ideal gas behavior). These predictions have been compared to activity coefficients and vapor-liquid equilibria by the UNIFAC model used as starting point. Moreover, VLE results have been compared with reported experimental data for the 
'individual mixture' approach. These reported data are obtained through TDE-NIST in AspenPlus version 10. These NIST database results are data validated by NIST and originate from Ortega and Espiau $^{51}$ for the ethanol-heptane mixture, from Heintz et al. ${ }^{52}$ for the 1-butanol-nonane mixture, from Rao et al. ${ }^{53}$ from the chloroform-toluene mixture, from Camero et al. ${ }^{54}$ for the MEKchloroform mixture and from Uslu et al. ${ }^{55}$ for the toluene-MEK mixture.

\section{FIRST VALIDATION OF THE PROPOSED METHOD (RESULTS AND DISCUSSION)}

In this section we will show that $g^{E}$ values can be directly related to $A^{E}$ from MIR spectra using a Partial Least Squares regression model. Secondly, we will show the constructed VLE diagrams based on these predicted $g^{E}$ values and will compare these to VLE diagrams obtained from the UNIFAC model. As stated earlier, generated Raman and NIR spectra have been used for the same purpose, but since preliminary analysis has shown that NIR and Raman spectroscopy results were similar to MIR results but generally slightly less accurate, the Raman and NIR results will not be shown. For illustration purposes RMSEP values obtained with the NIR, MIR and Raman for three individual mixtures are shown in Table S3 of the Supporting Information. Furthermore, it is expected that NMR or a combination of the spectroscopic techniques can be used to generate accurate Gibbs excess energy data as well.

\subsection{Proof-of-principle results: individual binary mixtures model}

The 30 MIR spectra measured for the different compositions of the MEK-toluene binary mixture and the MIR excess absorbance spectra calculated for this mixture are illustrated in Figure 4. An excess absorbance-Gibbs excess energy PLS model for this mixture has been created based on the $\mathrm{n}=21$ calibration spectra for this mixture (with compositions as listed in section 3.1 ) and the $g^{E}$ results generated from the 21 calibration compositions for this mixture using UNIFAC. In Figure 5 (left) predicted $g^{E}$ results for the $\mathrm{n}=9$ validation spectra (diamonds, with composition as listed in section 3.1) and the $n=21$ calibration spectra (circles) for this mixture based on the generated PLS model are compared with $g^{E}$ results for all compositions of the mixture obtained from UNIFAC ("actual Gibbs excess" in Fig. 5). Cross-validation has indicated that 3 LVs are required to create an accurate model. The RMSE of cross-validation (RMSECV) equals $4.54 \mathrm{~J} \cdot \mathrm{mol}^{-1}$ and the $R M S E$ of the validation set (RMSEP, where the $\mathrm{P}$ stands for Prediction) equals $2.97 \mathrm{~J} \cdot \mathrm{mol}^{-1}$. Based on these values and visual inspection of the results in Figure 5 (left) it can be concluded that it seems 
possible to predict $g^{E}$ values based on excess spectral (absorbance) data $A^{E}$ for the binary mixture MEK-toluene over the entire composition range.

The same applies for the binary mixtures chloroform-toluene (see Fig. 5 (middle)) and chloroformMEK (see Fig. 5 (right)), for which sufficiently low RMSECV and RMSEP have been obtained as well, taking the $g^{E}$ ranges for these mixtures into account. Furthermore, it is thus shown that individual $A^{E}$-g $g^{E}$ models are capable of accurately describing mixtures which show attractive molecular interaction (negative Gibbs excess energy, as observed for the chloroform-toluene and the chloroform-MEK mixtures) as well as mixtures for which repulsive non-ideality (positive Gibbs excess energy, as observed for the MEK-toluene mixture) plays a dominant role. The observed negative deviation from Raoult's law (negative Gibbs excess energy) for the chloroformtoluene mixture is caused by the interaction between the hydrogen of the chloroform molecule and the ring structure of the toluene molecule. ${ }^{56}$ For the chloroform-MEK mixture the negative deviation from Raoult's law is most likely caused by hydrogen bonding effects as well, since ketones are well-known proton acceptors, while chloroform is a proton donor. 

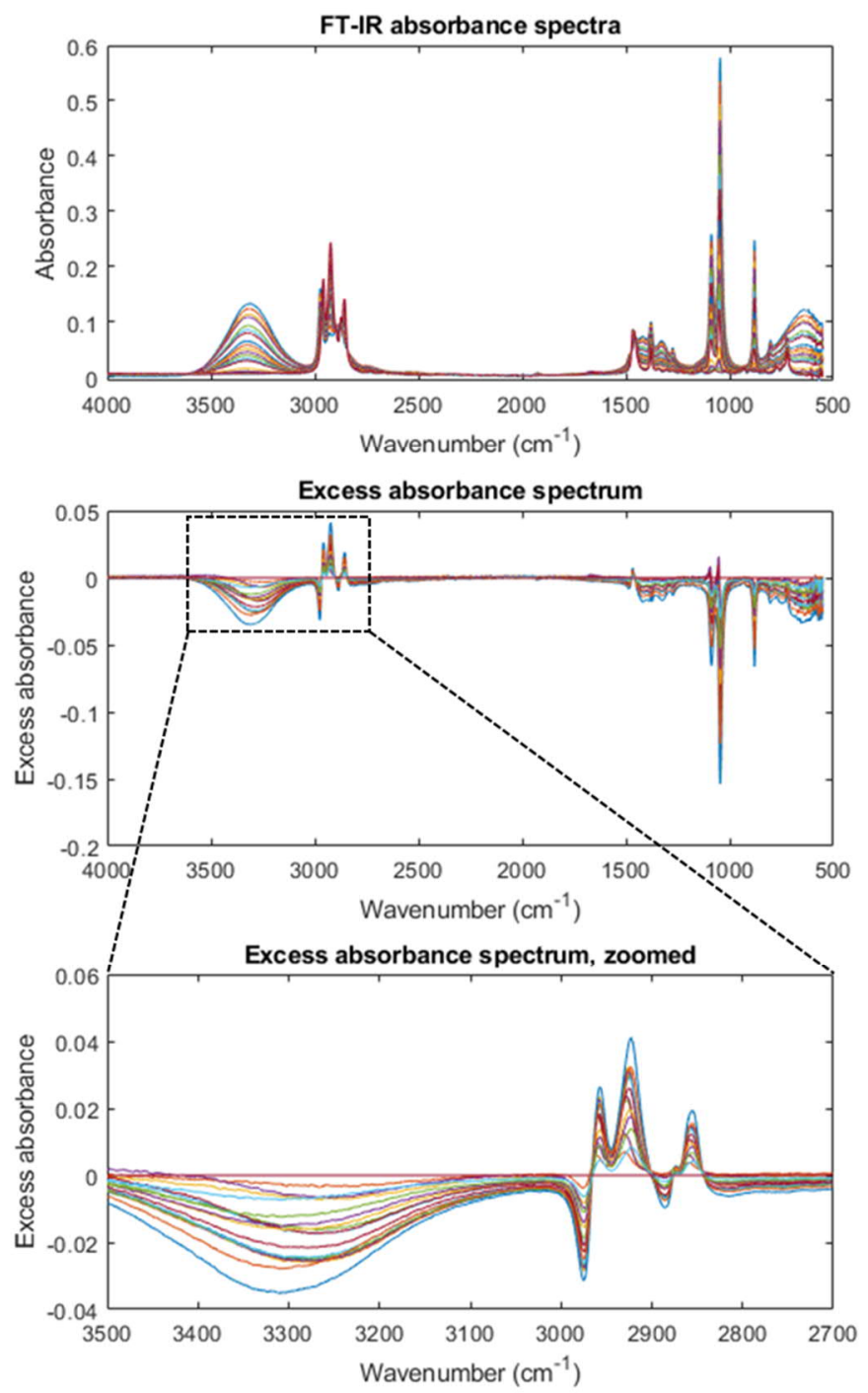

Figure 4. MIR absorbance (top), MIR excess absorbance (middle) spectra and zoomed MIR excess absorbance $\left(2700-3500 \mathrm{~cm}^{-1}\right.$ range, lower) for the MEK-toluene mixture for illustration purposes.
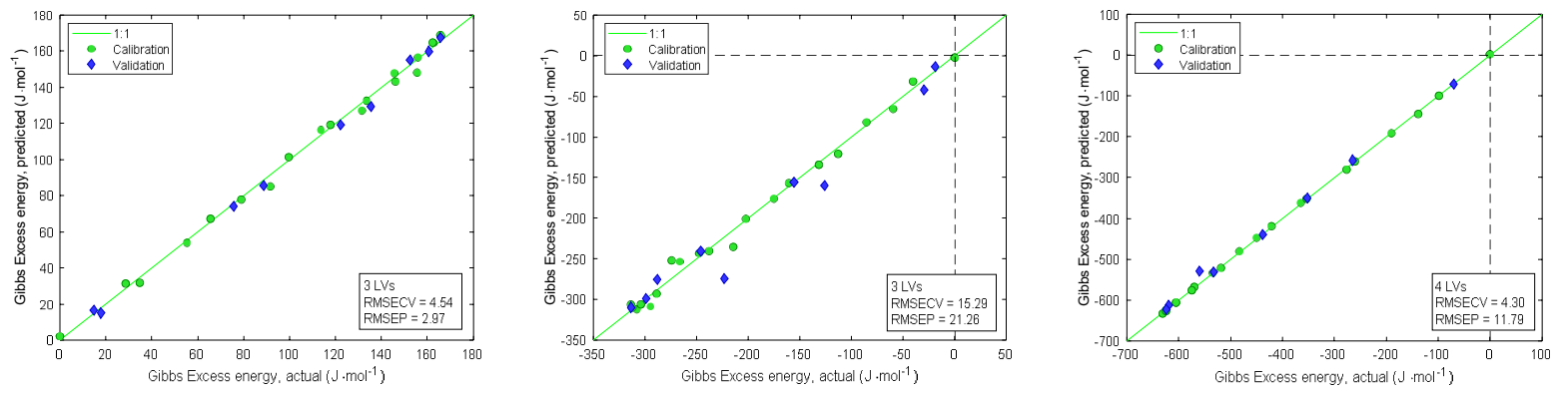

Figure 5. $A^{E}-g^{E}$ model prediction of the MEK-toluene mixture (left), chloroform-toluene mixture (middle) and chloroform-MEK mixture (right). The green circles show the prediction of the (21) calibration spectra for each individual mixture, and the blue diamond symbols show the prediction of the (9) validation spectra. 
For the heptane-ethanol mixture (see Fig. 6 (left)), even though proper calibration results have been obtained as indicated by the $R M S E C V=95.4 \mathrm{~J} \cdot \mathrm{mol}^{-1}$ (taking the $g^{E}$ range of $0-1600 \mathrm{~J} \cdot \mathrm{mol}^{-1}$ into account), the accuracy of the validation results was worse than for the other mixtures, as indicated by the $R M S E P=279 \mathrm{~J} \cdot \mathrm{mol}^{-1}$ for this mixture. The relatively poor validation for the heptane-ethanol mixture is not representative for all alkane-alcohol mixtures, as is shown for e.g. the nonane-1-butanol mixture for which accurate calibration and validation results have been obtained (see Fig. 6 (right) and the low $R M S E C V=76.2 \mathrm{~J} \cdot \mathrm{mol}^{-1}$ and $R M S E P=75.6 \mathrm{~J} \cdot \mathrm{mol}^{-1}$ in relation to the $g^{E}$ range of $\left.0-1300 \mathrm{~J} \cdot \mathrm{mol}^{-1}\right)$.
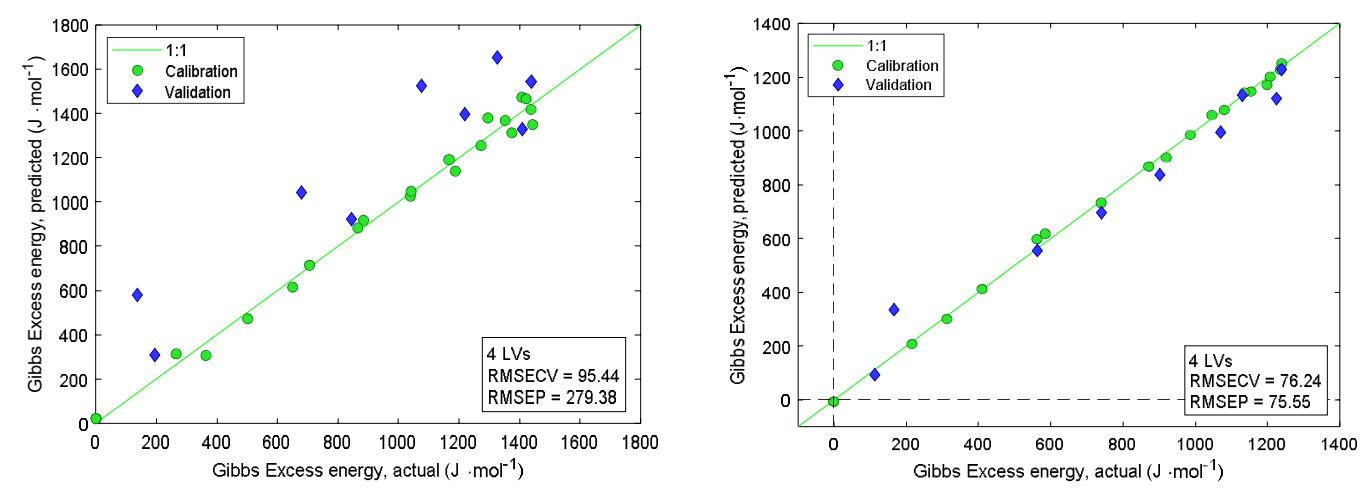

Figure 6. $A^{E}-g^{E}$ model prediction for the heptane-ethanol mixture (left) and the nonane-1-butanol mixture (right). The green circles show the prediction of the (21) calibration spectra, and the blue diamond symbols show the prediction of the (9) validation spectra.

As a next step of the comparison, the predictions have been compared in terms of representation of the phase equilibria. Following the described procedure (vide supra), the Txy-diagrams of a number of binary systems were constructed from the spectroscopic data as input. These predicted Txy-diagrams were compared with those predicted by UNIFAC and measured data points ${ }^{51-55}$ (see Fig. 7). For the binary mixtures consisting of chloroform, MEK and toluene, the predicted VLE data through the individual $A^{E}-g^{E}$ models correspond very well with the data from the UNIFAC model, and most importantly, with the measured literature data. It is notable that the VLE relates to a temperature range between $60^{\circ} \mathrm{C}$ and $120^{\circ} \mathrm{C}$, while the MIR absorbance was measured at room temperature. This shows for these systems that the individual $A^{E}-g^{E}$ models that were obtained from measurements and $g^{E}$ calibration data based on UNIFAC, both determined at room temperature only, can predict VLE behavior at different temperatures. We expect that this observation can be attributed to the use of the UNIQUAC model rather than to the methodology 
used. For improved accuracy, certainly for systems with a stronger temperature dependency, it is advantageous to collect the spectroscopic data at multiple temperatures.
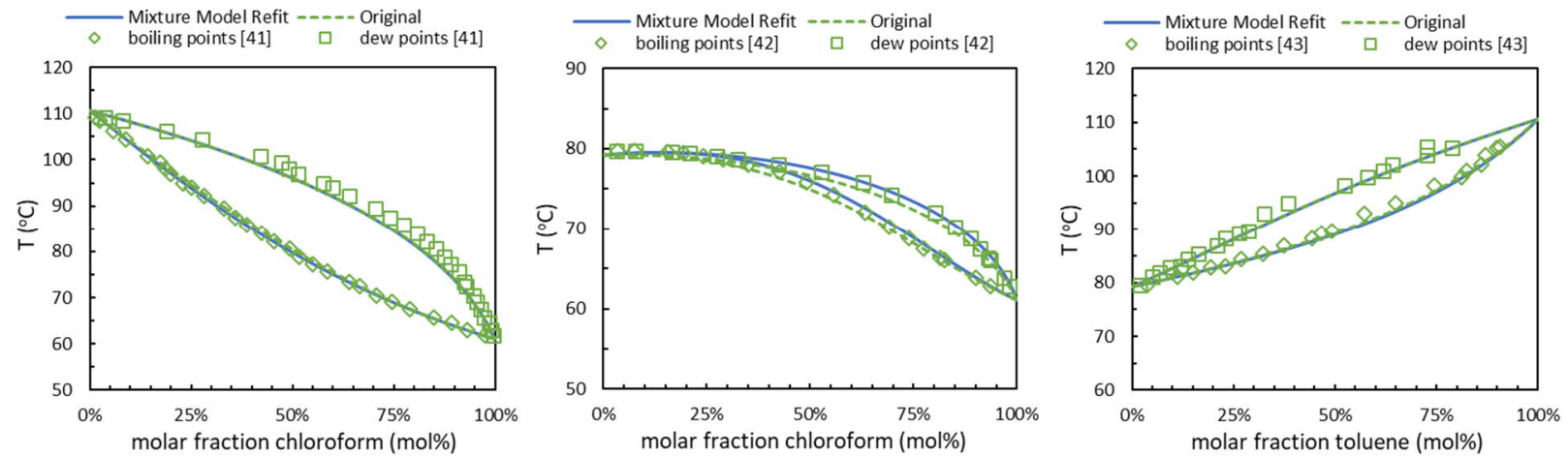

Figure 7. T-xy diagram at 1 bar for the chloroform-toluene mixture (left), MEK-chloroform mixture (middle) and toluene-MEK mixture (right). Blue solid lines represent predictions based on UNIQUAC binary interaction parameters obtained from the $A^{E}-g^{E}$ models, green dashed lines represent predictions based on UNIFAC, and symbols represent boiling point and dew point data from the NIST database originating from Rao et al. ${ }^{53}$, Camero et al. ${ }^{54}$ and Uslu et al. ${ }^{55}$, respectively.

The VLE diagrams for the heptane-ethanol mixture and the nonane-1-butanol mixture are shown in Figures 8 (left) and 8 (right), respectively. It can be concluded that for both mixtures a fair representation of the phase envelope is obtained, although a closer look at the nonane-1-butanol system shows that a liquid-liquid split (LLE) is predicted for part of the composition range which is not observed in practice ${ }^{52}$ nor predicted by UNIFAC. However, both systems show a strong positive deviation from ideality, even a homogeneous maximum temperature azeotrope. A slight overprediction of the non-ideality can result in the prediction of the (false) liquid-liquid split in this case. The prediction of the azeotrope composition and conditions is quite accurate, though a heterogeneous azeotrope is predicted instead of the homogeneous azeotrope.

Generally, the observed results show that, even though the prediction of the $g^{E}$ data from the family $A^{E}-g^{E}$ model is not always perfect, still accurate VLE data can be produced. These results show that for individual mixtures information from spectroscopic analysis (excess absorbance) can be used for prediction of VLE data for these individual mixtures, once a correlative model for such mixtures is established. UNIQUAC binary interaction parameters obtained from predicted Gibbs excess energy values for these individual mixtures are shown in the Supporting Information. 

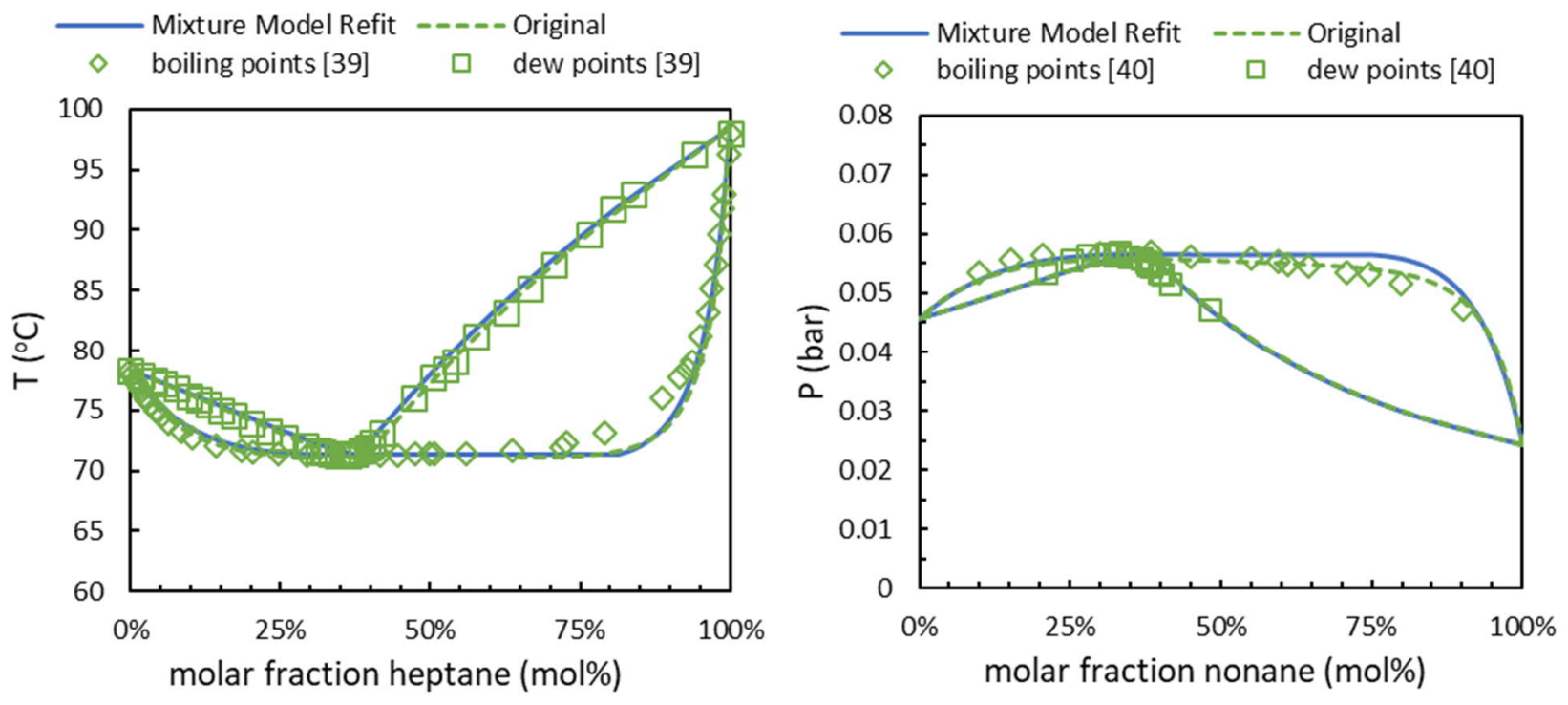

Figure 8. $\mathrm{T}$-xy diagram at $1 \mathrm{bar}$ for the heptane-ethanol (left) and $\mathrm{P}$-xy diagram at $\mathrm{T}=25^{\circ} \mathrm{C}$ for the nonane-1-butanol mixture (right). Blue solid lines represent predictions based on UNIQUAC binary interaction parameters obtained from the $A^{E}-g^{E}$ models, green dashed lines represent predictions based on UNIFAC, and symbols represent boiling point and dew point data from the NIST database originating from Ortega and Espiau ${ }^{51}$ and Heintz et al. ${ }^{52}$

\subsection{Proof of principle results: binary mixture family model and combined binary mixture families' model}

The alcohol-ketone binary mixture family consists of three alcohol members (ethanol, 1-butanol, and 1-hexanol) and three ketone members (MEK, 2-hexanone and 2-octanone), leading to nine binary mixtures in total (see Fig. 3 and Table 3). The corner points of the family have been used to set up the $A^{E}-g^{E}$ model. Hence, the 21 calibration compositions (see section 3.1 ) for the 4 binary mixtures consisting of the longest and shortest alcohols (ethanol and 1-hexanol) and the longest and shortest ketones (MEK and 2-octanone) within the alcohol-ketone family have been used to create the $A^{E}-g^{E}$ model (i.e. $\mathrm{n}=84$ excess MIR spectra based on the 21 calibration compositions for the 4 calibration mixtures). In Figure 9 (left) the $g^{E}$ results from the $A^{E}-g^{E}$ model for the calibration - and validation composition sets (see section 3.1) for these 4 mixtures are compared with $g^{E}$ results obtained from UNIFAC ( $g^{E}$ actual). After cross-validation it appeared that a $10 \mathrm{LV}$ model was required to create the PLS model. This increase in LVs compared to the individual mixtures' models is not surprising, since including more mixtures into a single model implies that more spectroscopic effects (i.e. LVs) need to be considered for accurate modeling. 
The performance of the generated $A^{E}$ - $g^{E}$ model is good as can be seen in Figure 9 (left) with RMSECV and RMSEP having about the same value $\left(16.8 \mathrm{~J} \cdot \mathrm{mol}^{-1}\right.$ and $20.7 \mathrm{~J} \cdot \mathrm{mol}^{-1}$, respectively) and being low compared to the range of $g^{E}$ values $\left(0-700 \mathrm{~J} \cdot \mathrm{mol}^{-1}\right)$ evaluated. Consequently, this is the first indication that the model can predict $g^{E}$ from excess absorbance spectra for the alcoholketone binary mixture family extremes that have been used in the calibration, but with compositions different from those used in the calibration. The obtained model is even capable of predicting $g^{E}$ for mixtures from the alcohol-ketone family that have not been used at all in the creation of the model (see Fig. 9 (right)). The accuracy of the predictions is somewhat worse (RMSEP has increased to $26.4 \mathrm{~J} \cdot \mathrm{mol}^{-1}$ ) than obtained for mixtures that were involved in the creation of the model (compare to Fig. 9 (left)), but still quite high considering the $g^{E}$ range (of 0 - $700 \mathrm{~J} \cdot \mathrm{mol}^{-1}$ ) evaluated. Thus, it has been clearly shown that an $A^{E}-g^{E}$ model generated based on a limited number of binary mixtures within a (alcohol-ketone) family, can predict the $g^{E}$ for other mixtures within that family accurately.
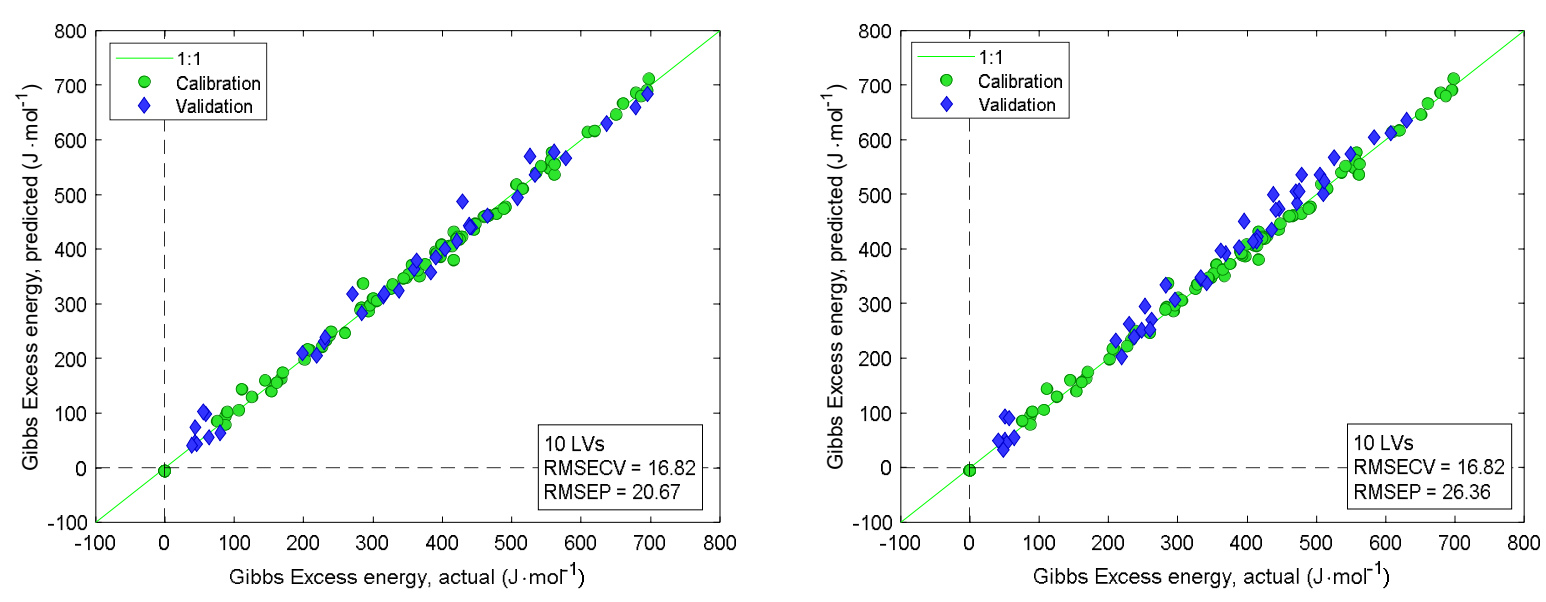

Figure 9. $g^{E}$ obtained from UNIFAC modeling (actual) compared to $g^{E}$ obtained from the $A^{E}-g^{E}$ model (predicted) for the alcohol-ketone family. The green circles represent predictions for the calibration set. The blue diamond symbols in the left graph represent the validation set of the same 4 mixtures, but at different compositions. In the right graph, the blue diamond symbols represent $g^{E}$ results of the mixtures from the alcohol-ketone family that have not been used in the calibration (creation) of the model with compositions that have not been used in the calibration of the model as well (i.e. the $\mathrm{n}=45$ spectra of the 5 other mixtures with 9 validation compositions each).

For the 4 mixtures of compounds that have been used in the construction of the model for the alkane-ketone family (the alkanes heptane and undecane, and the ketones MEK and 2-octanone), 
$g^{E}$ for compositions different from compositions used in the creation of the model can be predicted with sufficiently high accuracy $\left(R M S E C V=51.3 \mathrm{~J} \cdot \mathrm{mol}^{-1}\right.$ and $R M S E P=50.6 \mathrm{~J} \cdot \mathrm{mol}^{-1}$, see Fig. 10 (left)). Prediction of $g^{E}$ data for other alkane-ketone mixtures not included in the creation of the model is slightly less but still sufficiently accurate $\left(R M S E P=71.2 \mathrm{~J} \cdot \mathrm{mol}^{-1}\right.$, see Fig. 10 (right)) taking the $g^{E}$ range for this binary mixture family of $0-1100 \mathrm{~J} \cdot \mathrm{mol}^{-1}$ into account.

For the alkane-alcohol family the prediction of $g^{E}$ based on excess absorbance became less accurate. For the 4 mixtures of compounds from the alkane-alcohol family that have been used in the construction of the model (the alkanes heptane and undecane, and the alcohols ethanol and hexanol), $g^{E}$ for compositions different from those involved in the creation of the model have been predicted with reasonable accuracy $\left(R M S E C V=199 \mathrm{~J} \cdot \mathrm{mol}^{-1}\right.$ and $R M S E P=190 \mathrm{~J} \cdot \mathrm{mol}^{-1}$, see Fig. 11 (left)), taking into account the $g^{E}$ range of $0-2000 \mathrm{~J} \cdot \mathrm{mol}^{-1}$ for the alkane-alcohol binary mixture family. However, prediction of $g^{E}$ for other alkane-alcohol mixtures that were not involved in the construction of the model (using validation compositions only) is less accurate (RMSEP=305 $\mathrm{J} \cdot \mathrm{mol}^{-1}$, see Fig. 11 (right)).
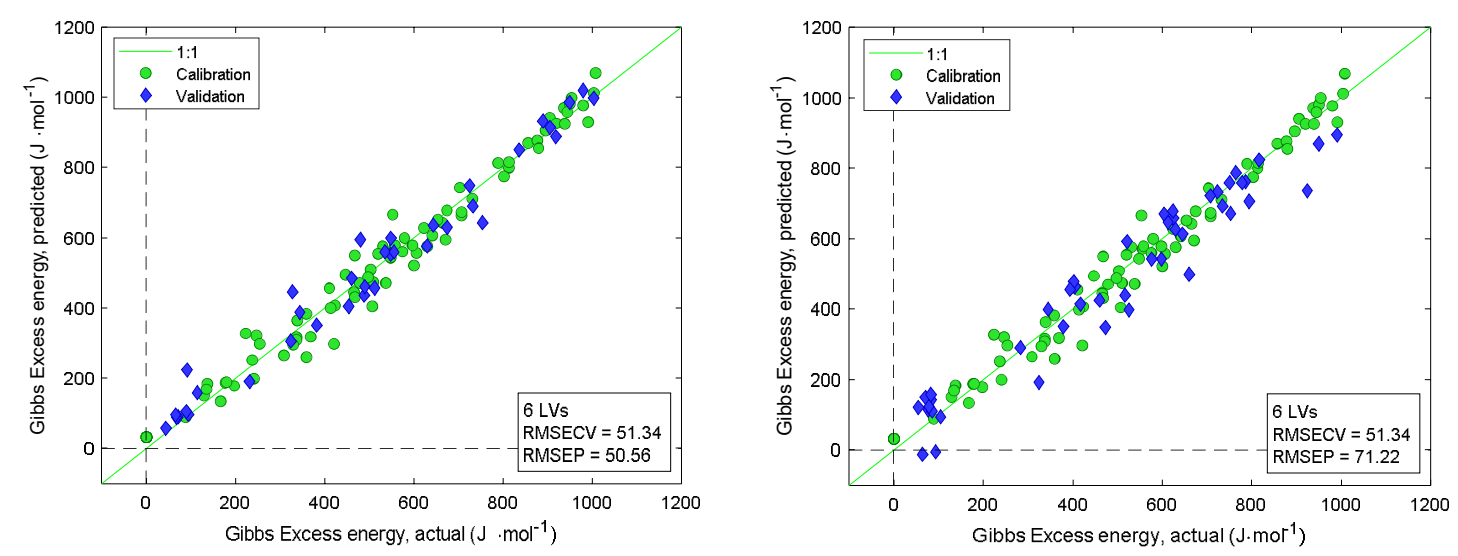

Figure 10. $g^{E}$ obtained from UNIFAC modeling (actual) compared to $g^{E}$ obtained from the $A^{E}-g^{E}$ model (predicted) for the alkane-ketone family. The green circles represent predictions of the calibration set. The blue diamond symbols in the left graph represent the validation set of the same mixtures at different composition. In the right graph, the blue diamond symbols represent $g^{E}$ results of the mixtures from the alkane-ketone family that have not been used in the calibration of the model with compositions that have not been used in the calibration of the model.

Based on the results for these three families it can be concluded that there indeed is a relation between $A^{E}$ from spectroscopic analysis and $g^{E}$, and that $g^{E}$ can be predicted within a binary 
mixture family. However, it is observed that in our proof of principle approach the $g^{E}$ based on the obtained $A^{E}$ from MIR seems to be less accurate when the binary mixture family becomes more non-ideal, as confirmed by the obtained Gibbs excess ranges for the different families (where the $g^{E}$ alcohol-ketone range $\left(0-700 \mathrm{~J} \cdot \mathrm{mol}^{-1}\right)<g^{E}$ alkane-ketone $\operatorname{range}\left(0-1100 \mathrm{~J} \cdot \mathrm{mol}^{-1}\right)<g^{E}$ alkane-alcohol range $(0$ $-2000 \mathrm{~J} \cdot \mathrm{mol}^{-1}$ ), see Figs. 9-11). There is a number of potential reasons for the observed deviations, basically in all steps of the methodology used in this study:

- The selected spectroscopic method (MIR) may be sub-optimal for highly non-ideal systems and another spectroscopic technique or a combination of techniques would provide better results.

- The spectroscopic measurements may be hampered by the occurrence of a second liquid phase (e.g. due to the presence of small quantities of water in the ethanol for the alkanealkanol family experiments).

- The current determination of the excess absorbance focusing primarily on peak amplitude taking peak shifts into account implicitly may not provide the most optimal description.

- The PLS model approach for the $A^{E}-g^{E}$ model may not provide an appropriate framework to representing the curve in this kind of strong non-ideality from spectral data, since it is more easily applicable to describe changes in amplitude rather than changes in peak shifts.

- The parameterization method used to derive UNIQUAC interaction parameters from Gibbs excess values obtained from the $A^{E}-g^{E}$ model. This is illustrated in Fig. 12 for the nonane1-butanol mixture, where the predicted $g^{E}$ data from the alkane-alcohol family $A^{E}$ - $g^{E}$ model describe the model input data from UNIFAC relatively well, but after deriving the UNIQUAC input parameters, the deviation between the resulting $g^{E}$ values from UNIQUAC and the UNIFAC $g^{E}$ used for calibration has increased.
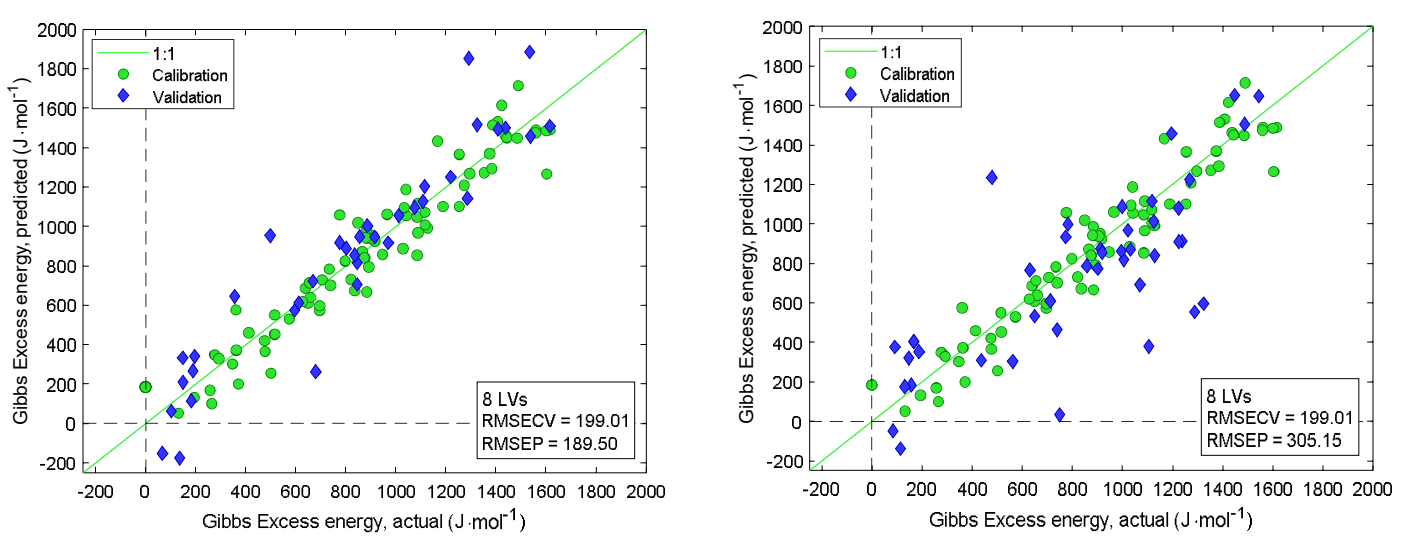

Figure 11. $g^{E}$ obtained from UNIFAC modeling (actual) compared to $g^{E}$ obtained from the $A^{E}-g^{E}$ model (predicted) for the alkane-alcohol family. The green circles represent predictions of the 
calibration set. The blue diamond symbols in the left graph represent the validation set of the same mixtures at different composition. In the right graph, the blue diamond symbols represent $g^{E}$ results of the mixtures from the alkane-alcohol family that have not been used in the calibration of the model with compositions that have not been used in the calibration of the model.

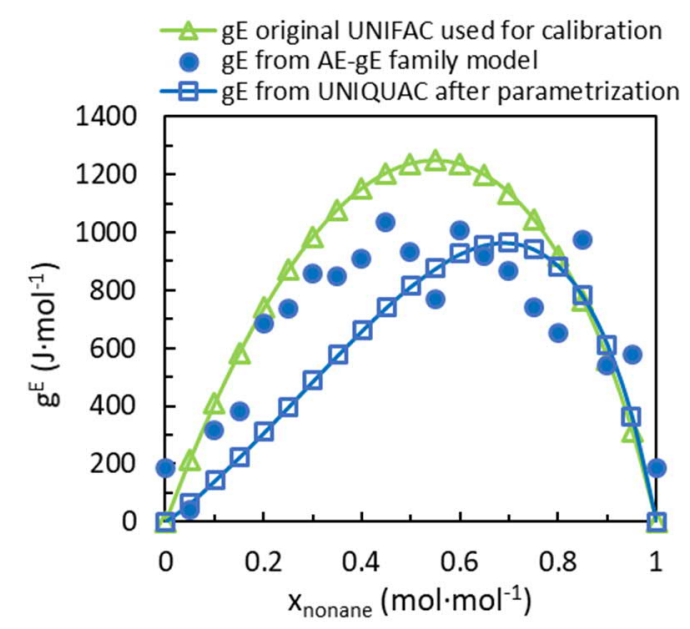

Figure 12. UNIFAC based $g^{E}$ values for the nonane-1-butanol mixture used in the calibration of the $A^{E}$ - $g^{E}$ alkane-alcohol family model compared with $g^{E}$ values predicted from the $A^{E}$ - $g^{E}$ alkanealcohol family model and the $g^{E}$ values obtained after UNIQUAC parameterization of the $g^{E}$ values predicted from the $A^{E}$ - $g^{E}$ alkane-alcohol family model.

Attempts to predict $g^{E}$ results for the alcohol-ketone \& alkane-ketone \& alkane-alcohol combined family based on a single calibration set using the 12 corner points consisting of the shortest and the longest alkane, ketone and alcohol were not (yet) successful (with $R M S E P=211 \mathrm{~J} \cdot \mathrm{mol}^{-1}$ ) as shown in Fig. 13 (left). However, the alcohol-ketone \& alkane-ketone combined $A^{E}$ - $g^{E}$ model which is based on a single calibration set using the 8 corner points consisting of the shortest and the longest alkane, ketone and alcohol, see Fig. 3 (below), can predict $g^{E}$ results accurately (with $\left(\right.$ RMSEP $\left.=52.3 \mathrm{~J} \cdot \mathrm{mol}^{-1}\right)$ as shown in Fig. 13 (right). The alcohol-ketone \& alkane-ketone combined family $A^{E}-g^{E}$ model is therefore able to predict $g^{E}$ values for mixtures that show hydrogen bonding behavior (alcohol-ketone mixtures) as well as for systems that do not show hydrogen bonding (alkane-ketone mixtures). Reasons why this approach does not work for the alcohol-ketone \& alkane-ketone \& alkane-alcohol combined family $A^{E}-g^{E}$ model (further including the alkanealcohol mixtures) is not completely clear and could give opportunities for further research. Furthermore, including more families with molecular interactions other than evaluated in this study may improve the predictability for combined families based on a single calibrated $A^{E}$ - $g^{E}$ model. 

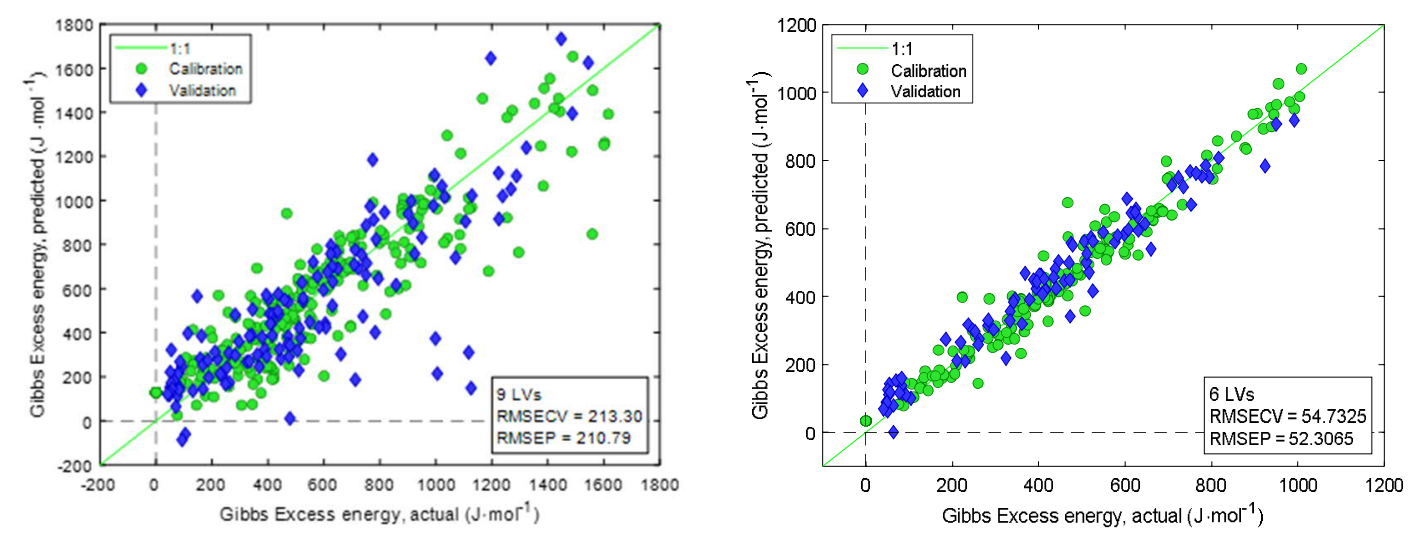

Figure 13. $g^{E}$ obtained from UNIFAC modeling (actual) compared to $g^{E}$ obtained from the $A^{E}$ - $g^{E}$ model (predicted) for the alkane-ketone \& alcohol-ketone \& alkane-alcohol combined family (left) and for the alkane-ketone \& alcohol-ketone combined family (right). The green circles represent predictions of the calibration set. The blue diamond symbols represent $g^{E}$ results of the mixtures from the combined family that have not been used in the calibration of the model with compositions that have not been used in the calibration of the model.

For process simulation tools based on thermodynamic models for e.g. separation processes, it is more important to have an accurate representation of the vapor-liquid equilibria (VLE), and by itself the quality of representation of the $g^{E}$ is less relevant. UNIQUAC binary interaction parameters obtained from predicted Gibbs excess energy values for the different binary families are shown in the Supporting Information. To assess the quality of the prediction of the vapor-liquid equilibria, isothermal pressure-composition ( $\mathrm{P}$-xy) diagrams have been constructed at $\mathrm{T}=25^{\circ} \mathrm{C}$ (the temperature of the isothermal spectroscopic measurements). As shown in Figure 14 the predicted Pxy-diagrams for the alkane-ketone family (see Fig. 3) correspond very well with those constructed with UNIFAC, not only for the binary systems involved in building the model, but also for the other family member systems that hence are fully predicted.

Though the P-xy phase envelope for the nonane-MEK mixture may suggest significant deviation from the original UNIFAC predictions (see Fig. 14b), it should be realized that these differences do not have a significant impact on the application, e.g. the design of a distillation column. As shown in Figure 15b, the differences in the $y$-x diagram are small, hence there is only a small error in the required number of stages for the separation of this mixture by distillation. 

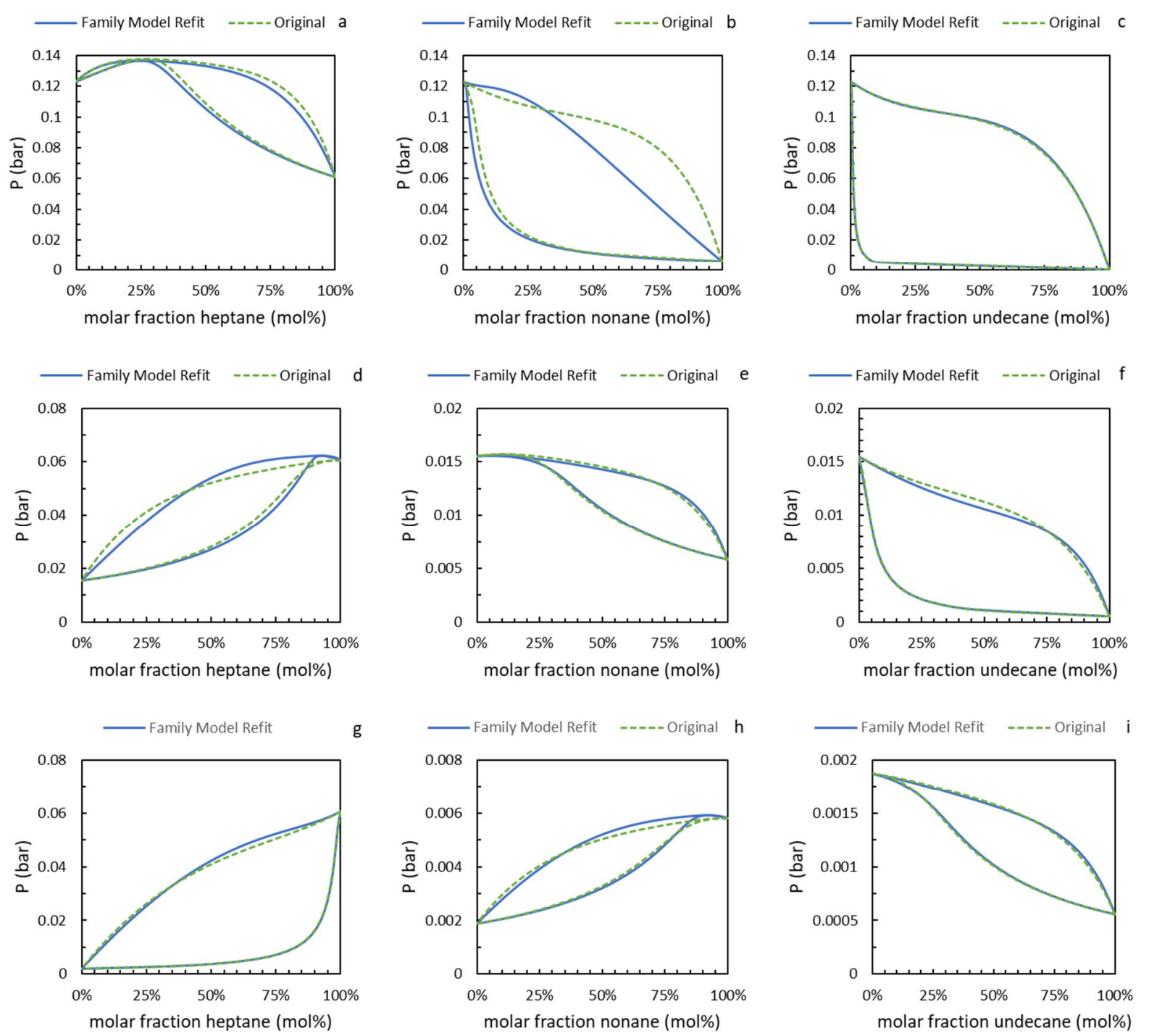

Figure 14. $\mathrm{P}$-xy diagrams at $\mathrm{T}=25^{\circ} \mathrm{C}$ for the alkane-ketone family (a for heptane-MEK, $\mathrm{b}$ for nonane-MEK, c for undecane-MEK, $\mathrm{d}$ for heptane-2-hexanone, e for nonane-2-hexanone, $\mathrm{f}$ for undecane-2-hexanone, $\mathrm{g}$ for heptane-2-octanone, $\mathrm{h}$ for nonane-2-octanone, and $\mathrm{i}$ for undecane-2octanone). Blue solid lines represent predictions based on the predicted $g^{E}$ results and green dashed lines present UNIFAC predictions

For the alcohol-ketone family the isothermal pressure-composition (P-xy) diagrams and the vaporliquid envelopes ( $\mathrm{y}$-x diagrams) for all mixtures are predicted even more accurately (results not shown), as can be expected from the more accurate prediction of the $g^{E}$ results (compare Fig. 9 and Fig. 10 for the alcohol-ketone and the alkane-ketone family, respectively). For the alcoholketone family, vapor-liquid envelopes generated via our methodology match the actual vaporliquid envelopes constructed by UNIFAC very well for all the mixtures within this family (results not shown). Hence for this class it is demonstrated again that the VLE behavior of binary mixtures that were not used in setting up the model, can be predicted from spectroscopic data. 
For the alkane-alcohol binary mixture family VLE predictions are clearly less accurate, as anticipated from the less perfect $g^{E}$ predictions (see Fig. 11). Especially the prediction of pressurecomposition profiles at $\mathrm{T}=25^{\circ} \mathrm{C}$ is relatively poor (see Fig. 16), even for mixtures that have been involved in the calibration of the model, such as the heptane-ethanol mixture (see Fig. 16a). In several cases the formation of a second liquid phase (liquid-liquid split) is observed indicated by the presence of a pressure independent composition range (see e.g. Fig. 17a-17c for the mixtures of ethanol with heptane, nonane and undecane, respectively). Predicted vapor-liquid composition diagrams, as used in determining the total number of stages required in distillation according to the McCabe-Thiele method, qualitatively shows the expected behavior and is sufficiently accurate for practical purposes (see Fig. 18). For a few binary mixtures azeotropes also are missing.
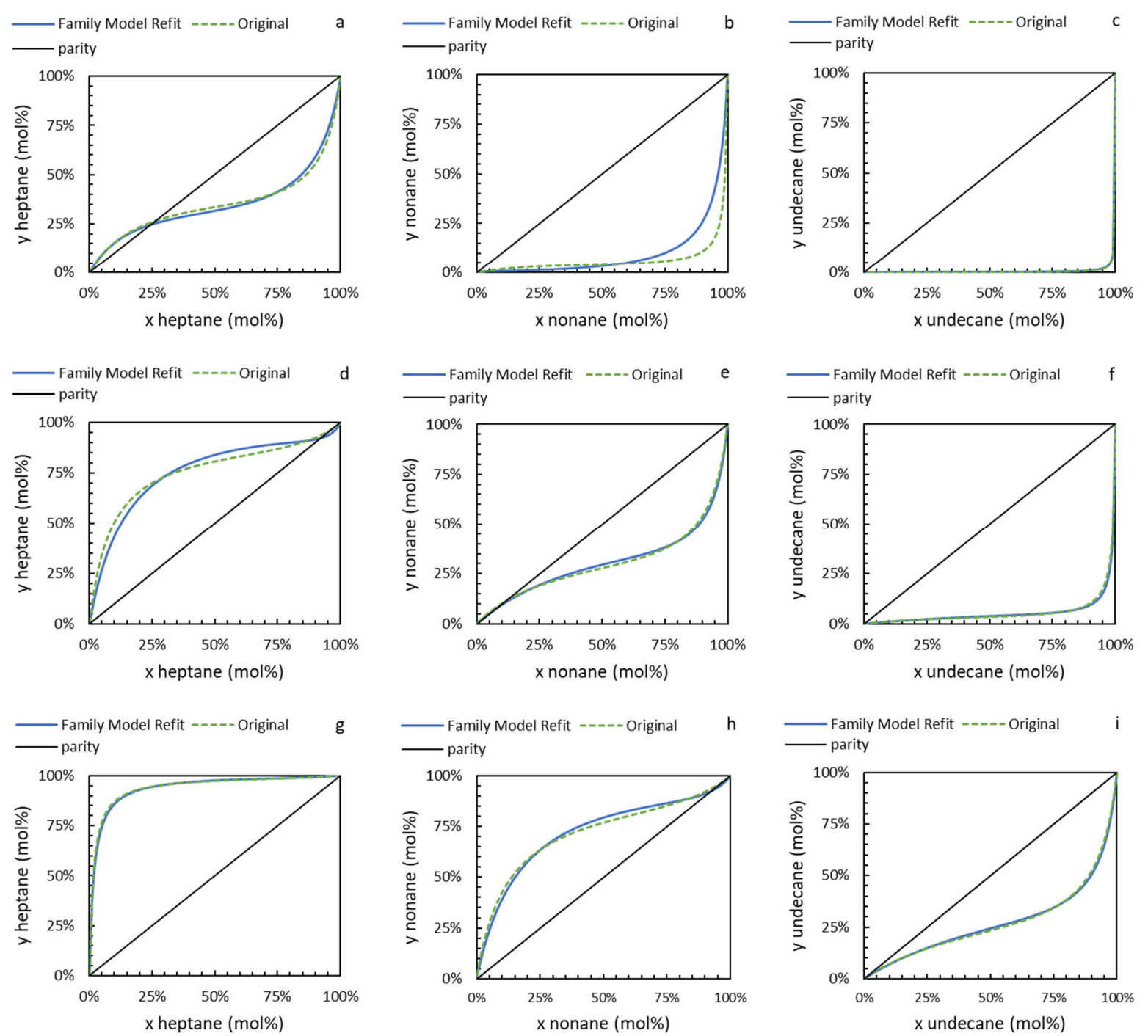

Figure 15. Vapor-liquid envelopes (y-x diagrams) at $\mathrm{T}=25^{\circ} \mathrm{C}$ for the alkane-ketone family (a for heptane-MEK, $\mathrm{b}$ for nonane-MEK, $\mathrm{c}$ for undecane-MEK, $\mathrm{d}$ for heptane-2-hexanone, e for nonane2-hexanone, $\mathrm{f}$ for undecane-2-hexanone, $\mathrm{g}$ for heptane-2-octanone, $\mathrm{h}$ for nonane-2-octanone, and i for undecane-2-octanone). Blue solid lines represent predictions based on the predicted $g^{E}$ results, green dashed lines present UNIFAC predictions and the black line represents equal molar composition of gas (y) and liquid (x) phase. 
Despite the relatively poor prediction of VLE for mixtures such as heptane-ethanol and nonane-1butanol in this family model approach, a proper VLE description could be obtained from the individual mixture models based on absorption excess data for these mixture as such (see Fig. 8 (left) and (right)), as shown earlier. This means that even though the model description for more highly non-ideal mixture families is (still) less accurate, it seems that even for these mixtures, excess absorbance data in MIR (and NIR and Raman spectroscopy) contain information that can be used to assess $g^{E}$. However, it is also clear that there is room for further improving the hypothesized methodology, for which only the first steps have been taken to fully proof this.
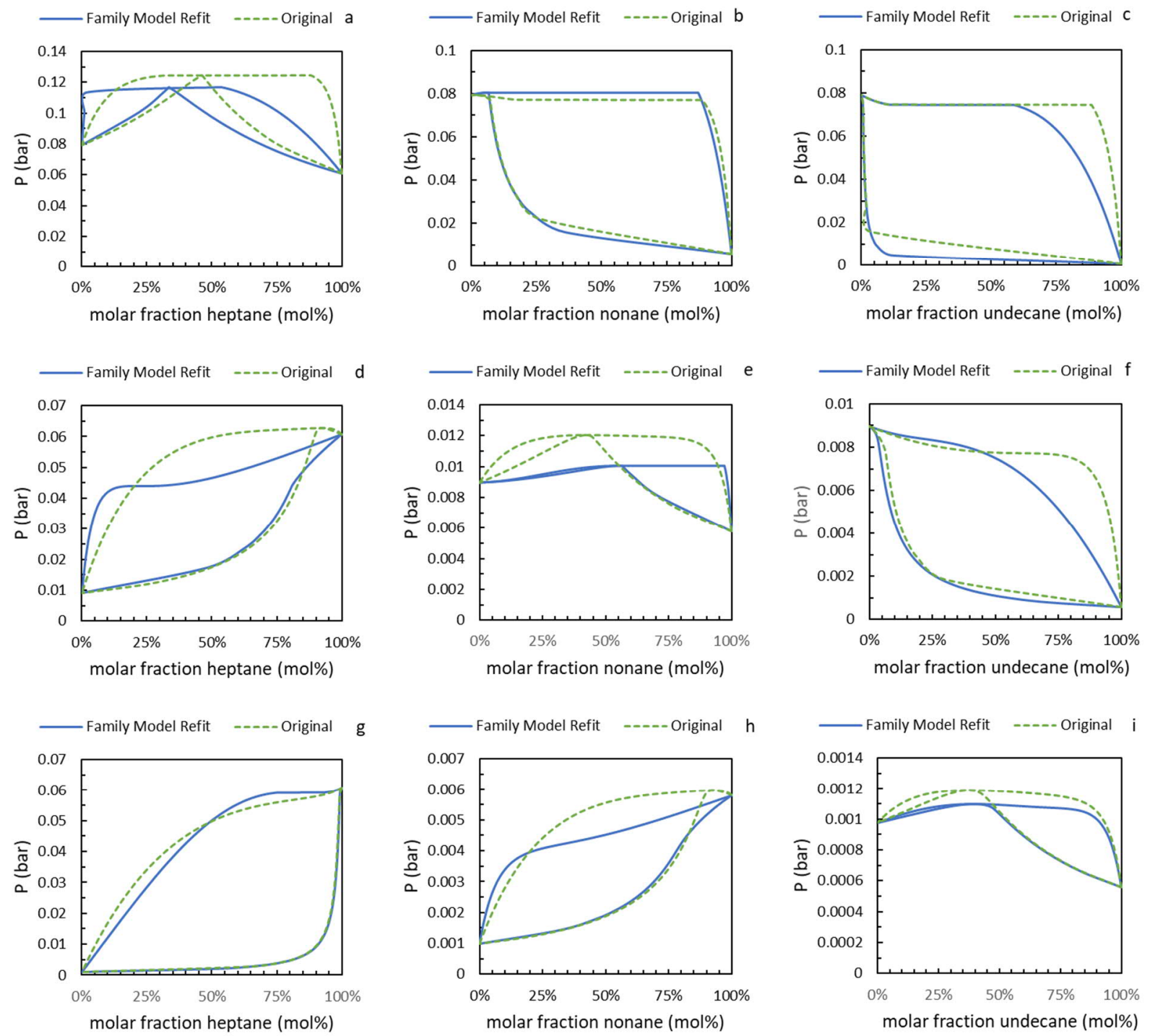

Figure 16. Pressure-composition $(\mathrm{P}-\mathrm{xy})$ diagrams at $\mathrm{T}=25^{\circ} \mathrm{C}$ for the alkane-alcohol family (a for heptane-ethanol, $\mathrm{b}$ for nonane-ethanol, c for undecane-ethanol, d for heptane-1-butanol, e for nonane-1-butanol, f for undecane-1-butanol, $g$ for heptane-1-hexanol, h for nonane-1-hexanol, and i for undecane-1-hexanol). Blue solid lines represent predictions based on the predicted $g^{E}$ results and green dashed lines present UNIFAC predictions 

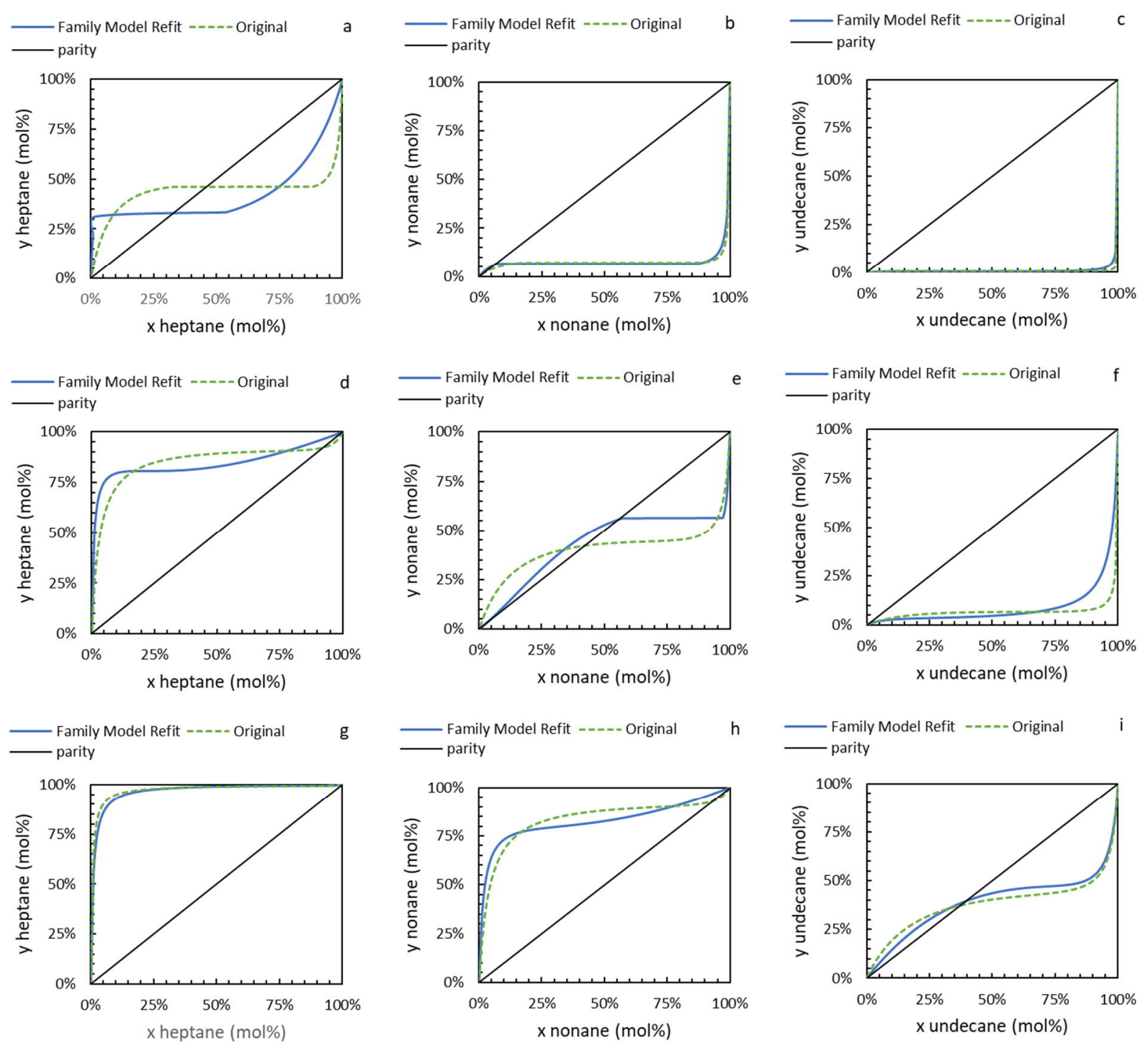

Figure 17. Vapor-liquid envelopes (y-x diagrams) at $\mathrm{T}=25^{\circ} \mathrm{C}$ for the alkane-alcohol family (a for heptane-ethanol, b for nonane-ethanol, c for undecane-ethanol, d for heptane-1-butanol, e for nonane-1-butanol, f for undecane-1-butanol, g for heptane-1-hexanol, h for nonane-1-hexanol, and i for undecane-1-hexanol). Blue solid lines represent predictions based on the predicted $g^{E}$ results, green dashed lines present UNIFAC predictions and the black line represents equal molar composition of gas (y) and liquid (x) phase.

\section{CONCLUSIONS}

We have demonstrated that spectroscopic methods such as infrared spectroscopy contain nonideality information for binary liquid mixtures. A framework has been proposed to predict activity coefficients from spectroscopic measurements as sole input, for which a first proof-of-principle has been given: a model relating the excess absorbance data to Gibbs excess energy data for individual mixtures could be used to successfully predict VLE data for these individual mixtures. The data-driven modeling approach by PLS was chosen to provide proof-of-principle. Although 
the MIR measurements used to create the model were only measured at room temperature and Gibbs excess data used for calibration of the models were obtained at room temperature as well, extrapolation of model results to much higher temperatures was found possible. The latter observation is likely due to the use of UNIQUAC with its built-in temperature dependence. This conclusion is valid for a wide range of mixtures, from mixtures showing negative deviation from Raoult's law (featuring strong attractive, often cross-interacting molecular forces such as hydrogen bonding indicated by negative Gibbs excess energy data) to mixtures showing positive deviation from Raoult's law (implying that the interactions between same type of molecules are stronger than those between unlike molecules, indicated by positive Gibbs excess energy data). Furthermore, and more importantly, within a family of component mixtures, such as an alcoholketone family (consisting of different alcohol-ketone binary mixture members) and an alkaneketone family (consisting of different alkane-ketone binary mixture members), Gibbs excess energy data and VLE diagrams for all members of the mixture family can be predicted from an excess absorbance-Gibbs excess energy model that was generated using only a limited amount of family mixture members. Therefore, even though Gibbs excess energy data are still needed for calibration of the model, the amount of Gibbs excess energy data required to determine VLE for all mixtures within a family can be reduced significantly. Though the prediction based on this family approach became less accurate for the alkane-alcohol family, a first proof of principle was given for the methodology and the framework. So far it has not been possible to predict Gibbs excess data from excess absorbance from a single calibrated model based on all families combined (alkane-ketone \& alcohol-ketone \& alkane-alcohol). A single model for the combined alkaneketone \& alcohol-ketone families was able to predict Gibbs excess energy and VLE data properly for alkane-ketone and alcohol-ketone mixtures. Therefore, even though the first steps in proving the proposed methodology have been taken, several optimization options are foreseen and needed for each of the various steps of the hypothesized framework. Further validation of the proposed methodology is deemed necessary, especially to create a better physical relation between the spectroscopic signal and the excess energy function. This could be subject of further study.

\section{AUTHOR INFORMATION}

\section{Corresponding author}

*E-mail: gerrald.bargeman@nouryon.com 


\section{ORCID}

Gerrald Bargeman: 0000-0002-4035-9064

Notes

The authors declare no competing financial interest.

\section{Author contributions}

The manuscript was written through contributions of all authors. Antoon J.B. ten Kate: Conceptualization, Methodology, Software, Validation, Formal analysis, Investigation, Resources, Data Curation, Writing - Original Draft, Writing - Review \& Editing, Visualization, Supervision, Project administration; Jan Gerretzen: Methodology, Software, Validation, Formal analysis, Investigation, Resources, Data Curation, Writing - Original Draft, Writing - Review \& Editing, Visualization, Supervision; Henk-Jan van Manen: Methodology, Validation, Resources, Data Curation, Writing - Review \& Editing, Supervision, Funding acquisition; Georgios M. Kontogeorgis: Validation, Writing - Original Draft, Writing - Review \& Editing; Gerrald Bargeman: Conceptualization, Methodology, Validation, Resources, Data Curation, Writing Original Draft, Writing - Review \& Editing, Visualization, Supervision, Project administration, Funding acquisition. All authors have given approval to the final version of the manuscript.

\section{ACKNOWLEDGEMENTS}

Martijn Smout, René van Egdom, Ali Ghamati, and Georgios Stavropoulos are kindly thanked for their involvement in preparing, measuring and analyzing all mixtures. Paul Verwer, Martina Costa Reis, Michele Mattei, Jacob Bart, and Tony Davies are thanked for providing valuable insights during discussions.

\section{ABBREVIATIONS}

\section{Nomenclature}

$\begin{array}{lll}\text { Symbol } & \text { Description } & \text { Unit } \\ a & \text { Intercept in relation between } \boldsymbol{y} \text { and } \boldsymbol{X} & \left(\mathrm{J} \cdot \mathrm{mol}^{-1}\right) \\ A & \text { Absorbance } & \end{array}$


the vector of regression coefficients (one for each wave $\left(\mathrm{J} \cdot \mathrm{mol}^{-1}\right.$ )

number in the excess absorbance spectra) in the relation

between $\boldsymbol{y}$ and $\boldsymbol{X}$

$B_{i j}$

Binary interaction parameter $\mathrm{B}$ describing the interaction between components $i$ and $j$

$B_{j i}$

Binary interaction parameter $\mathrm{B}$ describing the interaction

between components $\mathrm{j}$ and $\mathrm{i}$

$H$

$f$

$g$

$M$

$n$

$q_{i}$

$Q_{k}$

$r_{i}$

$R_{k}$

RMSE

RMSECV

RMSEP

$S$

$v_{k}^{i}$

$\boldsymbol{X}$

$x_{i}$

$y$

$y$

$\hat{y}$

$y_{i}$

Greek
Enthalpy

Helmholtz energy

Gibbs energy

Property of actual solution

Number of spectra

Surface area parameter for component $\mathrm{i}$

Group area parameter

Volume parameter for component i

Group volume parameter

Root Mean Square Error

Root Mean Square Error for Cross Validation

Root Mean Square Error for Prediction

Entropy

Number of groups $\mathrm{k}$ in molecule $\mathrm{i}$

The matrix of excess absorbance spectra $\left(A^{E}\right)$

Molar based liquid fraction for component $\mathrm{i}$

the vector of Gibbs excess energy $\left(g^{E}\right)$ values

True value for Gibss excess $g^{E}$

Predicted value for Gibss excess $g^{E}$

Molar based gas fraction for component $\mathrm{i}$

Coordination number (taken equal to 10)
$\left(\mathrm{J} \cdot \mathrm{mol}^{-1}\right)$

$\left(\mathrm{J} \cdot \mathrm{mol}^{-1}\right)$

$\left(\mathrm{J} \cdot \mathrm{mol}^{-1}\right)$

$(-)$

$(-)$

$(-)$

$(-)$

$(-)$

$(-)$

$(-)$

$\left(\mathrm{J} \cdot \mathrm{mol}^{-1} \cdot \mathrm{K}^{-1}\right)$

(-)

$\left(\mathrm{J} \cdot \mathrm{mol}^{-1}\right)$

$\left(\mathrm{J} \cdot \mathrm{mol}^{-1}\right)$

$\left(\mathrm{J} \cdot \mathrm{mol}^{-1}\right)$

$(-)$

(-) 


$\begin{array}{lll}\theta_{i} & \text { Area fraction of component i } & (-) \\ \varphi_{i} & \text { Volume fraction of component i } & (-) \\ \tau_{i j} & \text { Interaction parameter for component i related to component } & (-) \\ & \mathrm{j}\end{array}$

\section{Superscript}

$\begin{array}{ll}E & \text { Excess value } \\ \text { id } & \text { For ideal solution } \\ \text { pure } & \text { Of pure component }\end{array}$

\section{Subscript}

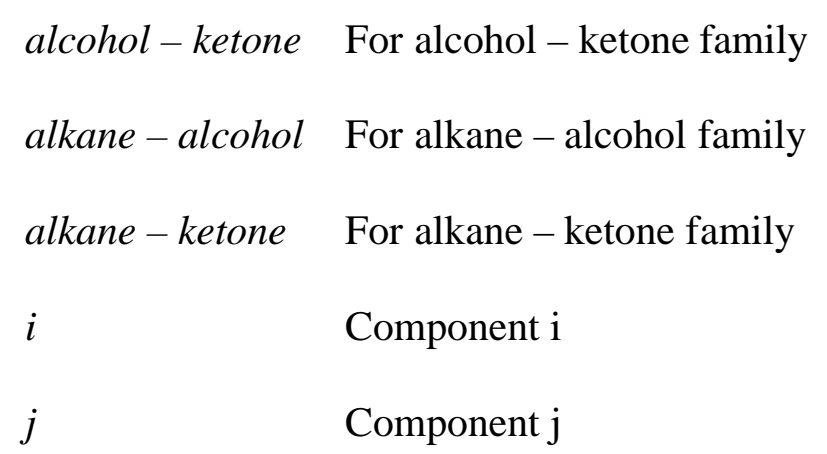

\section{Abbreviation}

ATR

Attenuated Total Reflectance

EOS

Equation of State

HTE

High throughput experimentation

ITC Isothermal calorimetry

LV Latent Variable

MIR Mid-InfraRed

NIR Near-InfraRed

NMR Nuclear Magnetic Resonance

PLS Partial Least Squares

PCA Principal Component Analysis

UV-vis Ultraviolet - visible

VLE Vapor Liquid Equilibrium 


\section{ASSOCIATED CONTENT}

\section{Supporting Information}

The Supporting Information shows UNIQUAC binary interaction parameters obtained for individual mixtures and mixture family mixtures, and an example for comparison of mid-infrared, near infrared and Raman spectroscopy results (PDF)

This information is available free of charge via the Internet at http://pubs.acs.org/

\section{REFERENCES}

(1) Hendriks, E.; Kontogeorgis, G. M.; Dohrn, R.; de Hemptinne, J-Ch.; Economou, I. G.; Zilnik, L. F.; Vesovic, V. Industrial Requirements for Thermodynamics and Transport Properties. Ind. Eng. Chem. Res. 2010, 49, 11131-11141.

(2) Cobos, A.; Sikorski, P.; González, J. A.; Królikowski, M.; Hofman, T. Solid-liquid equilibria and excess enthalpies in binary mixtures of acetophenone with some aliphatic amides. $J$. Mol. Liq. 2019, 296, 112058.

(3) Dorn, U. Enders, S. Heat of mixing and liquid-liquid-equilibrium of water + polypropylene glycol (PPG) with different molecular weights and water + propylene glycol dimethyl ether. Fluid Phase Equilib. 2016, 424, 58-67.

(4) Sprakel, L. M. J.; Schuur, B. Improving understanding of solvent effects on intermolecular interactions in reactive liquid-liquid extraction with isothermal titration calorimetry and molecular modeling. J. Ind. Eng. Chem. 2019, 72, 364-373.

(5) Sprakel, L. M. J.; Schuur, B. Molecular design and engineering for affinity separation processes using isothermal titration calorimetry (ITC) and molecular modeling (MM). $J$. Mol. Liq. 2019, 283, 312-324.

(6) Sprakel, L. M. J.; Kamphuis, P.; Nikolova, A. L.; Keijsper, D. J.; Schuur, B. Solvent selection for extractive distillation processes to separate close-boiling polar systems. Chem. Eng. Res. Des. 2019, 144, 123-134.

(7) Sprakel, L. M. J.; Keijsper, D. J.; Nikolova, A. L.; Schuur, B. Predicting solvent effects on relative volatility behavior in extractive distillation using isothermal titration calorimetry (ITC) and molecular modeling (MM). Chem. Eng. Sci. 2019, 210, 115203. 
(8) Sprakel, L. M. J. Schuur, B. Thermal activity in affinity separation techniques such as liquidliquid extraction analyzed by isothermal titration calorimetry and accuracy analysis of the technique in the molar concentration domain. Ind. Eng. Chem. Res. 2018, 57, 12574-12582.

(9) Jones, B. H.; Huber, C. J.; Spector, I. C.; Tabet, A. M.; Butler, R. A. L.; Hang, Y.; Massari, A. M. Correlating solvent dynamics and chemical reaction rates using binary solvent mixtures and two-dimensional infrared spectroscopy. J. Chem. Phys. 2015, 142, 212441.

(10) Da Silva, D. C.; Ricken, I.; Silva, M. A. D. R.; Machado V. G. Solute-solvent and solventsolvent interactions in the preferential solvation of Brooker's merocyanine in binary solvent mixtures. J. Phys. Org. Chem. 2002, 15, 420-427.

(11) Asprion, N.; Hasse, H.; Maurer, G. Thermodynamic and IR spectroscopic studies of solutions with simultaneous association and solvation. Fluid Phase Equilib. 2003, 208, 2531.

(12) Asprion, N.; Hasse, H.; Maurer, G. Application of IR-spectroscopy in thermodynamic investigations of associating solutions. Fluid Phase Equilib. 2003, 205, 195-214.

(13) Jirasek, F.; Burger, J.; Hasse, H. Method for estimating activity coefficients of target components in poorly specified mixtures. Ind. Eng. Chem. Res. 2018, 57, 7310-7313.

(14) Sassa, Y.; Katayama, T. Investigation for thermodynamic properties of alcoholic solutions by InfraRed Spectroscopic Study. J. Chem. Eng. J. 1973, 6, 31-37.

(15) Sassa, Y.; Katayama, T. Investigation for excess Gibbs free energies of alcoholic solutions by InfraRed Spectroscopic Study. Effects of association between unlike molecules. J. Chem. Eng. J. 1974, 7, 1-7.

(16) Ma, L.; Li, H.; Wang, C.; Xu, Y.; Han, S. Prediction of Vapor-Liquid Equilibria Data for CH Band Shifts of Raman Spectra and Activity Coefficients at Infinite Dilution in Some Aqueous Solutions. Ind. Eng. Chem. Res. 2005, 44, 6883-6887.

(17) Xiao, Z. H. U.; Jia, Y. A. O.; Haoran, L. I.; Shijun, H. A. N. Prediction of Vapor-Liquid Equilibrium Data from C-H Band Shift of IR Spectra in Some Binary Systems. Chin. J. Chem. Eng. 2007, 15, 97-101, https://doi.org/10.1016/S1004-9541(07)60040-8.

(18) Xu, Y.; Li, H.; Wang, C.; Ma., L.; Han, S. Prediction of vapor-liquid equilibria of alcoholhydrocarbon systems by ${ }^{1} \mathrm{H}$ NMR and activity coefficients at infinite dilution. Ind. Eng. Chem. Res. 2005, 44, 408-415. 
(19) Xu, Y.; Yao, J.; Yao, P.; Li, H.; Han, S. Prediction of vapor-liquid equilibria of alcoholhydrocarbon systems by ${ }^{1} \mathrm{H}$ NMR and azeotropic point. Chin. J. Chem. Eng. 2010, 18, 455461.

(20) Xu, Y.; Qian, W.; Gao, Q; Li, H. Prediction of vapor-liquid equilibria of alcohol+hydrocarbon systems by ${ }^{1} \mathrm{H}$ NMR spectroscopy. Chem. Eng. Sci. 2012, 74, $211-$ 218.

(21) Wilson, G. M. Vapor-liquid equilibrium. XI. A new expression for the excess free energy of mixing. J. Am. Chem. Soc. 1964, 86, 127-130.

(22) Deng, D.; Li, H.; Yao, J.; Han, S. Simple local composition model for ${ }^{1} \mathrm{H}$ NMR chemical shift of mixtures. Chem. Phys. Lett. 2003, 376, 125-129.

(23) Zhu, Q.; Moggridge, G. D.; Dalton, T.; Cooper, J.; Mantle, M. D.; Gladden, L. F.; D'Agostino, C. Letter to the Editor Assessing the use of NMR chemical shifts for prediction of VLE in non-ideal binary liquid mixtures. Chem. Eng. Sci. 2014, 119, 331-333.

(24) Xu, Y. Letter to the Editor Reply to the comments on "Assessing the use of NMR chemical shifts for prediction of VLE in non-ideal binary liquid mixtures" by C. D'Agostino. Chem. Eng. Sci. 2014, 119, 318.

(25) Li, Q.; Wang, N.; Zhou, Q.; Sun, S.; Yu, Z. Excess Infrared Absorption Spectroscopy and Its Applications in the Studies of Hydrogen Bonds in Alcohol-Containing Binary Mixtures. Appl. Spectrosc. 2008, 62, 166-170.

(26) Bergonzi, I.; Mercury, L.; Brubach, J. -B.; Roy, P. Gibbs free energy of liquid water derived from infrared measurements. Phys. Chem. Chem. Phys. 2014, 16, 24830.

(27) Israelachvili, J. N. Intermolecular and Surface Forces, Part I: The forces between atoms and molecules (Third Edition); Academic Press: Amsterdam, 2011.

(28) Shafique, M.; Hussain, S.; Asif, S.; Pradhan, V.; Farooqui, M. Thermodynamic Characteristics of Solvents: A Review. Res. J. Chem. Sci. 2013, 3, 98-104.

(29) González, J. A.; Domanska, U.; Lachwa, J. Thermodynamics of binary mixtures containing a very strongly polar compound — Part 3: DISQUAC characterization of NMP + organic solvent mixtures. Can. J. Chem. 2003, 81, 1451-1461.

(30) Rani M.; Maken, S. Thermodynamics of molecular interactions in binary mixtures containing associated liquids. Korean J. Chem. Eng. 2013, 30, 1636-1643. 
(31) Alvarez, V. H.; Mattedi, S.; Martin-Pastor, M.; Aznar, M.; Iglesias, M. Thermophysical properties of binary mixtures of \{ionic liquid 2-hydroxy ethylammonium acetate+(water, methanol, or ethanol)\}. J. Chem. Thermodyn. 2011, 43, 997-1010.

(32) Smith, J. M.; Van Ness, H. C.; Abbott, M. M. Introduction to Chemical Engineering Thermodynamics; McGraw-Hill: Boston, 2007; pp 709.

(33) Kiaee, H.; Rostami, A. A.; Farmanzadeh, D., Excess properties and spectroscopic studies for a binary system of polyethylene glycol 200 and N-methyl-2-pyrrolidone at different temperatures. J. Mol. Liq. 2017, 231, 242-248.

(34) Naejus R.; Lemordant, D.; Coudert, R.; Willmann, P. Excess thermodynamic properties of binary mixtures containing linear or cyclic carbonates as solvents at the temperatures 298.15 K and 315.15 K. J. Chem. Thermodyn. 1997, 29, 1503-1515.

(35) Corsetti, S.; Zehentbauer, F. M.; McGloin, D.; Kiefer, J. Characterization of gasoline/ethanol blends by infrared and excess infrared spectroscopy, Fuel, 2015, 141, 136-142.

(36) Wülfert, F.; Kok, W. Th.; Smilde, A. K. Influence of Temperature on Vibrational Spectra and Consequences for the Predictive Ability of Multivariate Models, Anal. Chem., 1998, 70, 1761-1767.

(37) Witjes, H.; Pepers, M.; Melssen, W. J.; Buydens, L. M. C. Modelling phase shifts, peak shifts and peak width variations in spectral data sets: its value in multivariate data analysis, Analitica Chimica Acta, 2001, 432, 113-124.

(38) Vandeginste, B.G.M.; Massart, D.L.; Buydens, L.M.C.; de Jong, S.; Lewi, P.J.; SmeyersVerbeke J. Handbook of Chemometrics and Qualimetrics Part B, Elsevier: Amsterdam; 1998; pp 324-336.

(39) Wold, S.; Sjöström, M.; Eriksson, L. PLS-regression: a basic tool of chemometrics. Chemometrics and intelligent laboratory systems 2001, 58, 109-130.

(40) Geladi, P.; Kowalski, B. R. Partial least-squares regression: a tutorial. Analytica Chimica Acta, 1986, 185, 1-17.

(41) Fuller, M. P.; Ritter G. L.; Draper, C. S. Partial least-squares quantitative analysis of infrared spectroscopic data. Part I: Algorithm implementation. Applied Spectroscopy, 1988, 42, 217 227. 
(42) Fischer, K.; Gmehling, J. Further development, status and results of the PSRK method for the prediction of vapor-liquid equilibria and gas solubilities. Fluid Phase Equilibria 1995, $112,1-22$

(43) Gmehling, J.; Kolbe, B.; Kleiber, M.; Rarey, J.; Chemical Thermodynamics for Process Simulation; John Wiley \& Sons: Weinheim, 2012.

(44) Fredenslund, Aa.; Gmehling, J.; Rasmussen P.; Vapor-Liquid Equilibria using UNIFAC; Elsevier: Amsterdam, 1977.

(45) Fredenslund, Aa.; Jones, R.L.; Prausnitz, J.M. Group contribution estimation of activity coefficients in nonideal liquid mixtures. AIChE J. 1975, 21, 1086-1099.

(46) Hansen, H.K.; Rasmussen, P.; Fredenslund, Aa.; Schiller, M.; Gmehling, J. Vapor-Liquid Equilibria by UNIFAC Group Contribution. 5 Revision and Extension. Ind. Eng. Chem. Res. 1991, 30, 2352-2355.

(47) Wittig, R.; Lohmann, J.; Gmehling, J. Vapor-Liquid Equilibria by UNIFAC Group Contribution. 6. Revision and Extension. Ind. Eng. Chem. Res. 2003, 42, 183-188.

(48) Basso, R. C.; da Silva, C. A. S.; de Oliveira Sousa, C.; de Almeida Meirelles, A. J.; Batista, E. A. C. LLE experimental data, thermodynamic modeling and sensitivity analysis in the ethyl biodiesel from macauba pulp oil settling step. Bioresource Technol. 2013, 131, 468475.

(49) Abrams, D. S.; Prausnitz, J. M. Statistical thermodynamics of liquid mixtures: a new expression for the excess gibbs energy of partly or completely miscible systems. AIChE J. 1975, 21, 116-128.

(50) Bondi, A.A. Physical Properties of Molecular Crystals, Liquids and Gases; Wiley: New York, 1968.

(51) Ortega, J.; Espiau, F. A New Correlation Method for Vapor-Liquid Equilibria and Excess Enthalpies for Nonideal Solutions Using a Genetic Algorithm. Application to Ethanol + an n-Alkane Mixtures. Ind. Eng. Chem. Res. 2003, 42, 4978-4992.

(52) Heintz, A.; Dolch, E.; Lichtenthaler, R. N. New experimental VLE-data for alkanol/alkane mixtures and their description by an extended real association (ERAS) model. Fluid Phase Equilib. 1986, 27, 61-79. 
(53) Rao, M. R.; Sitapathy, R.; Anjaneyulu, N. S. R.; Raju, G. J. V. J.; Rao, C. V. J.; Studies in vapour-liquid equilibria-- binary systems: acetone + toluene and chloroform + toluene. Sci. Ind. Res. 1956, Sect. B 15, 556-560.

(54) Camero, C. G.; Bhethanabotla, V. R.; Campbell, S. W. J. Total Pressure Measurements for Chloroform + Acetone + 2-Butanone at 303.15 K. Chem. Eng. Data 1995, 40, 210-213.

(55) Uslu, H.; Hasdemir, I. M.; Sipahi, N. Isobaric (vapour-liquid) equilibria data for the binary systems (toluene+acetic acid) and (toluene+methyl ethyl ketone) at atmospheric pressure. Phys. Chem. Liq. 2009, 47, 614-625.

(56) Tokuhiro T.; Woo, K.W.; Proton spin-lattice relaxation in the chloroform-toluene liquid system. A contribution to the elucidation of dynamic local structure. J. Phys. Chem. 1976, $80,733-740$. 
For Table of Contents Only

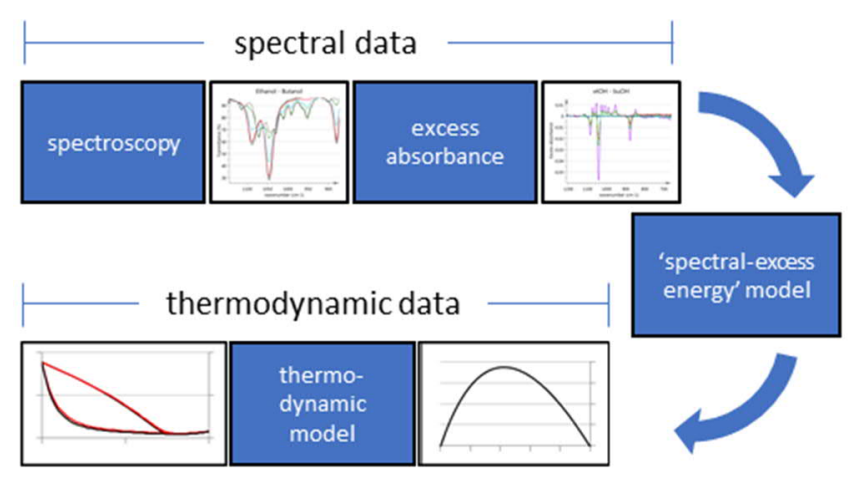

